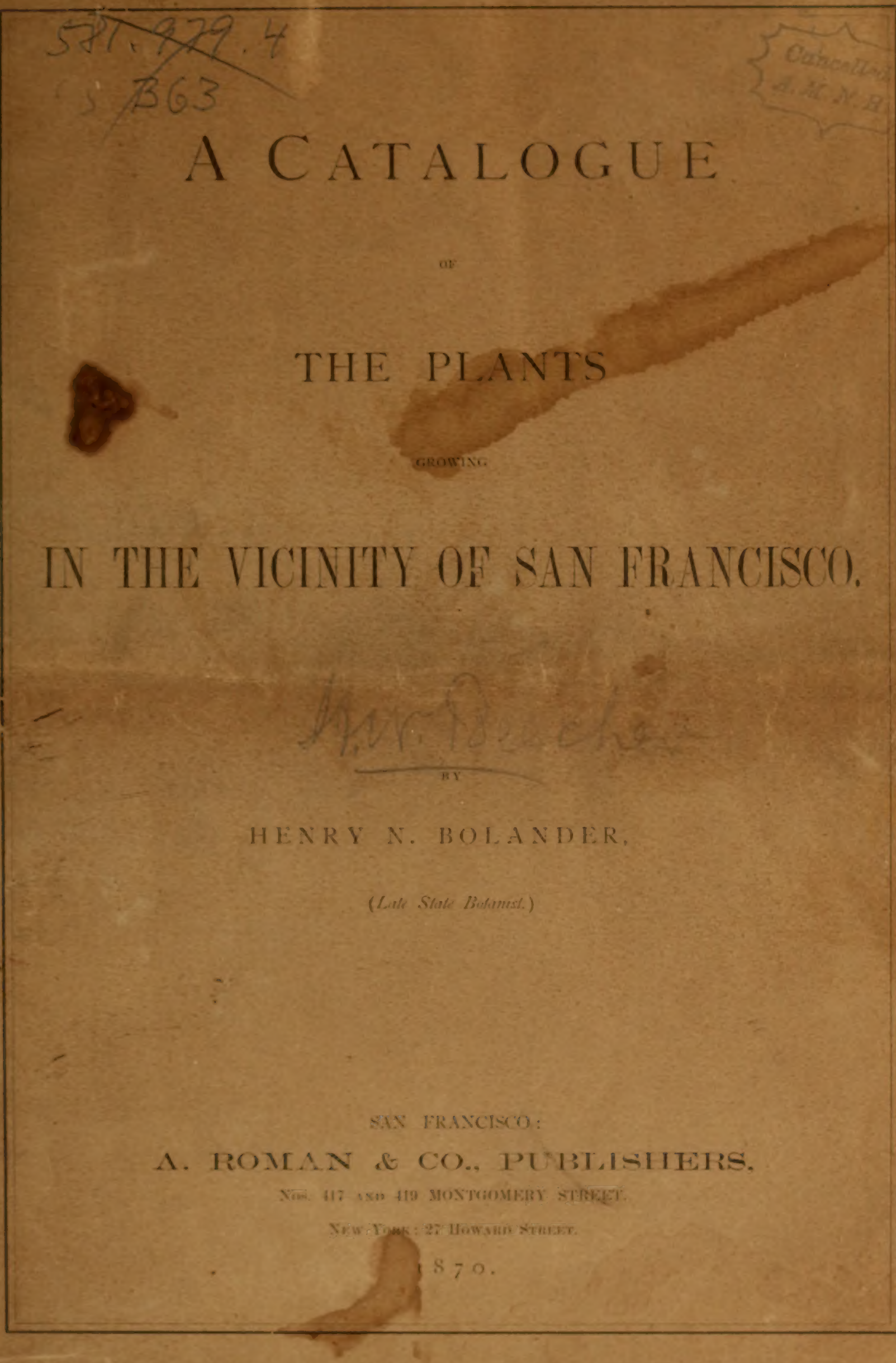



A CataloguE

OF

THE PLANTS

RROWINe:

\section{IN THE VICINITY OF SAN FRANCISCO.}

HENRY N. BOLANDER,

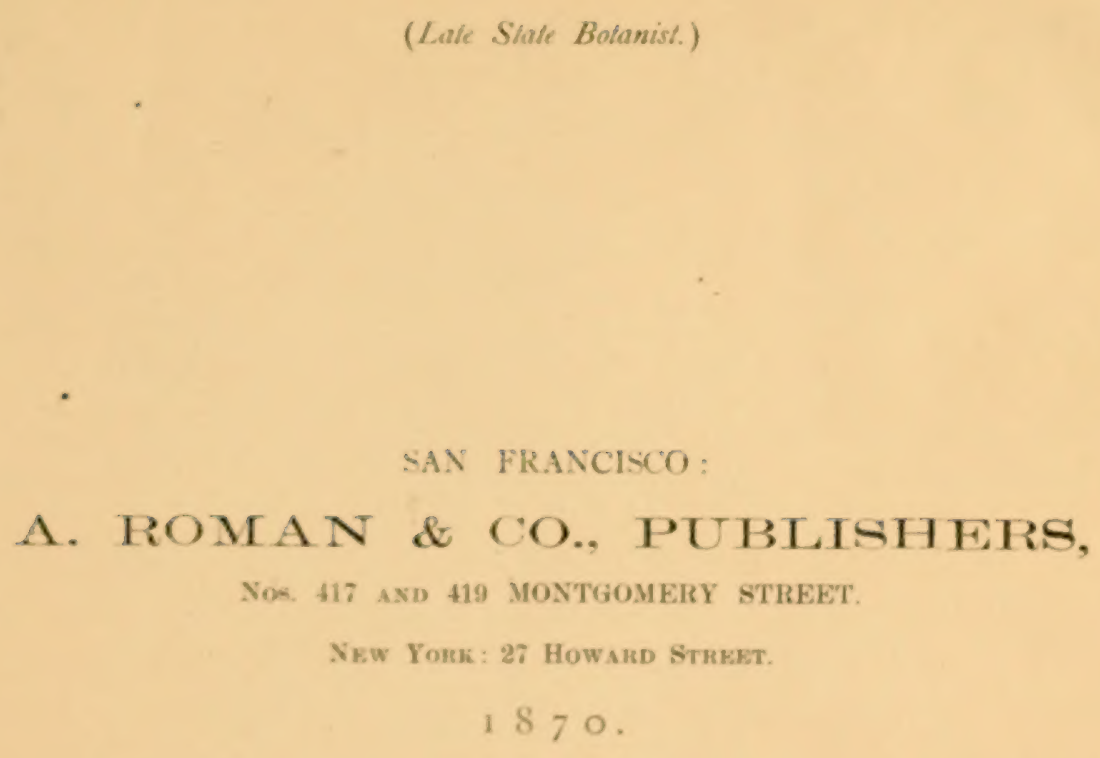

(Lale Siate Bolanist.)

SAN FRANCISCO:

A. ROMAN \& CO., PUBLISHERS,

Nos. 417 AND 419 MONTGOMERY STREET.

New Yom: : 27 Howahd STREkT.

1870 . 


$$
\begin{aligned}
& \text { Q } 194 \\
& . B 6 \\
& c .2
\end{aligned}
$$




\section{A CATALOGUE}

O $\mathrm{F}$ Т $\mathrm{F}$

\section{PLants Growing IN THE VicintTy of SAN Francisco.}

By II. N. Botander, lato State Botanist.

This catalogue contains the names of very nearly all the phaenogamous and cryptogamous plants found about a hundred miles north and south of San Franciseo, and as far east as Mount Diablo. Some plants were omitted (especially the shrubl y oaks) since it was impossible to determine their proper speeifie names. The two important families, the Fungi and Algæ have been entirely omitted; no one as far as I know, has made them a subject of investigation and study on this coast. The ferns, mosses, liverworts and lichens were studied by the very best authorities in America.

I take great pleasure in stating that our resident botanists, Dr. A. Kellogg and G. H. Bloomer, have largely assisted me in this undertaking, in fact, this catalogue is rather the result of our conjoint labors.

Neither the time of flowering has been mentioned, being so nearly alike for all, nor the underlying rock, since we have also in this respect so great a uniformity. The common metamorphic sandstone underlies nearly the whole region, except a few small patches of limestone and bituminous slate near Pescadero and Monterey. The influence of the underlying rock is apparently of less note, than the mechanical and physiesl properties of the subsoil and the distance from the ocean. From the ocean to Mount Diablo, one easily distinguishes three distinct floral regions; the immediate coast, the redwood and the parklands. Every one of these floral regions has its peculiar types and forms. Having no text book on our Flora, this catalogue may be of some service to those who are interested in the study of botany.

\section{Latrs Nasrs.}

Clematis, I., .

C. Iasiantha, Nutr.,

C. ligusticifolia, Benth. Anemone, I.,

A. nemorosa, L., var.,

Thaluetrum, Tourn.,

Th. Fendleri, Englm.,

Th. dioicum, I.,

Romonendus, L.

R. aquatilis, L.,

R. Californicus, Benth.

R. repens, $\mathrm{I}$.,

R. hebecarpus, Hook \& Arn.

R. muricatus, $\mathrm{I}_{\text {s, }}$

R. acris, $\mathbf{L}_{\text {. }}$,

R. reptans, L.,

Myonerus, Dill.,

M. minimus, I.,

Aquilegia, Tourn.,

A. Californica, Gray, .

\section{Ranunculacere.}

Evglisu Names.

Natural II anitat.

Virgin's Bower.

River banks

Windflower.

Meadow-Rue.

Redwood

Meadows

Buttercup

Ponds

Meadows

$" 6$

Around Shrubs

Streets of S. F.

Meadows

Mouse-Tail.

Columbine.
Sandy Places

Meadows and Hedges 
Latin Names.

Delphinium, Tourn.

D. Californicum, Torr and Gray.,

D. Menziesii, D. C.,

D. patens, Benth.,

D. simplex, Dougl.,

D. varigatum, Torr \& Gray.

D. decorum, Fisch. \& Mey.,

D. nudicaule, Torr. \& Gray., Actoa, L.,

A. spicata, L., var arguta.,
Englisi Names.

Larkspur.

Yellow Larkspur.

Baneberry.
Natural Habitat.

Meadows

Hillsides

"6

"

Hillsides

Plains

Gravelly Places

Redwoods

\section{Berberidaceae.}

Berberis, Is., .

Barberry.

Hillsides

B. pinnata, Lag.,

Vancouveria, Dene.,

V. hexandra, Morr \& Dee., Achlys, D. C.,

A. triphylla, Hook.,

Redwoods

Redwoods

Nymphaeacea. (Water Lily Fam.)

Brasenia, Schrebr.,

B. peltata, Pursh.

Nuphar, Smith.,

N. advena., Ait.,

Eschscholtzia, Cham.

E. Californica, Cham.

E. cæspitosa, Lindl.

Dendromecon, Benth.,

D. rigidum, Benth.

Meconopsis, Vig.

M. heterophylla, Benth.

Platystemon, Benth.,

P. Calitornicum, Benth., .

Platystigma, Benth.,

P. lineare, Benth.,

P. Californicum Torr, (Meconella)

Water Shield.

Pond Lily.

Clear Lake

Yellow Pond Lily.

Ponds

Papaveraceze. (Poppy Fam.)

Calif. Poppy.

Meadows

Dry Hillsides

Thickets

Sandy Soil

Sandy Soil

Mission Hills

Fumariaceae. (Fumitory Fam.)

Dutchman's Breeches.

Dicentra, Bork.

D. formosa, D. C

D. chrysantha, Hook.,

Cheiranthus, R. Br.,

Cruciferae. (Mustard Fam.)

Ch. capitatus, Dougl.,

Nasturtium, R. Br.,

N. officinale, R. Br.,

$\mathrm{N}$. curvisiliqua, Nutt.,

Barbarea, R. Br.,

B. vulgaris, R. B.,
Water Cress.

Winter Cress.
Shady Places

Dry Hillsides 
Lativ Names,

Exglisu NAMEs,

Streptanthus, Nutt.,

H. Breweri, Gray.,

H. glandulosus, Hook.

H. hispidus, Gray

H. flavescens, Hook., .

H. procerus, Brewer.,

Turritis, Dill.,

T. perfoliats, Lam., (T. glabra L.,)

Arabis, L.

A. blepharophylia, Hook and Arn., .

A. arcuatus, Nutt.,

Cardamine, $\mathbf{L}$,

C. angulata, Hook.,

C. oligosperma, Nutt.,

C. pauciseeta, Beuth.,

C. hirsuta, L. var.,

Sirymbrium, L,

S. officinale, Scop.,

S. deflexum, Harvey.,

S. pygmaeum, Nutt., .

S. csnescens, Nutt.,

Erysimum, L.,

E. asperum, D. C.

E. Arkansanum, Nutt.,

Brassica, Tourn.

B. alba, L.,

B. nigra, L.,

Smebiera, Poir.,

S. didyma, Pers.

Lepedium, R. Br.,

L. Menziesii, D. C.,

I. Californicum, Nutt.,

L. nitidum, Nutt.

L. oxyearpum, Torr and Gray

L. latipes, Hook.

Capsella, Vent.,

C. Bursa-Pastoris, Moench.,

Thysomacarpus, Hook.

Th. elegans, Fisch and Mey.,

Th. radians, Benth.,

Th. oblongifolius, Nutt.

Th. pulchellus, Fisch and Mey.

Th. pusillus, Hooks,

Raphanus, L.

R. raphanistrum, I.,

Fiola, $\mathrm{I}$.

V. canina, Rich,

V. Nuttallii, Pursho,

V. peduneulats, Gray and Torro,

V. glabella, Nutt.,

V. ocellata, Torr and Gray, (Canadensis ?)

V. sarmentosa, Dough,

Tower Mustard

Rock Cress.

Bitter Cress.

Hedge Mustard.

Treacle Mustard.

White Mustard.

Black Mustard.

Wart Cress.

Pepper-grass.

Shepherd's Purse.

Radish.
Blue Violet.

Yellow Violet.

Naturar, Habitat.

Monte del Diablo

Sandy Places

Monte del Diablo

Hillsides

Sandy Soil

Meadows

Mission Hills

Rocks

Meadows

Meadows

Redwoods

Fields

Hillsides

Hillsides

Hillsides

Fiélds

Fields

Banks

Fields

Fields

Oakland Hills

Fields

Meadows

Meadows

Hillsides

Fields

Violacere. (Violet Family.)

Moist Places

Gravelly Hillsides

Mission Hills

Redwoods

Redwoods

Redwoods 
Latin Names.

V. ehrysantha, Hook,

V. lobata, Benth.,
Englisir Names.

Natural Habitat.

Clay Soil

Woods

\section{Cistaceze. (Rock-Rose Family.)}

Rock-rose

Helianthemum, Tour.,

H. scoparium, Nutt.,
Hillsides

\section{Mypericaceze. (St. John's Wort Family.)}

Hypericum, L.,

H. anagalloides, Cham. Schl.,

H. Scouleri. Hook.,

Silene, L.,

S. Californica, Durand,

S. Drummondii, Hook.

S. Bolanderi, Gray.

S. Antirrhina, L.

S. Gallica, L.,

S. quinquevulnera, $\mathrm{L}$.

S. Douglasii, Hook.

Arenaria, L.,

A. Douglasii, Fenzl., .

A. Californica, Brew.

A. tenella, Nutt.

Stellaria, L.,

H. media, Smith.

H. crassifolia, Ehrh.

H. littoralis. Torr and Gray.

Cerastium, L.,

C. oblongifolium, Torr,

Sagina, L.,

S. procumbens, $\mathrm{L}$.

Spergularia, Pers.,

S. rubra, Presl.,

Spergula, L.,

S. arvensis, L.,

Paronychia, Tourn.,

P. ramosissima, D. C.
St. John's Wort.

Moist places.

Banks.

\section{Caryophyllaceze. (Pink Family.)}

Catchfly.

\section{Hillsides}

Open woods.

Fields.

Sandwort.

Sandy soil.

Chickweed.

Common Chickweed,

Fields.

Mouse-ear Chickweed.

Pearlwort

Banks.

Sand Spurrey.

Spurrey.

Corn Spurrey,

Whitlow Wort.

Portulacaceze. (Purslane Family.)

Calandrinia, H., B. and K.

C. Menziesii, Hook.,

Claytonia, L.,

C. perfoliata, Don.,

C. exigua, Torr and Gray.

C. Siberica, L.,

A. aquatica, Nutt.,

C. parvifolia, Mocino.

C. Menziesii. (?)

C. linearis, Dougl.,

Montia, L.,

M. fontana, L.,
Spring Beauty.

Meadows.

Fields and thickets.

Wet places.

Swamps.

Wet places.

In water. 
Lativ Names.

Evolisu Names.

Naturar. Habitat.

Lenoisia, Pursh.

Rocky places.

L. rediviva, Pu

S. umbellata, Torr.,

Sandy soil.

Malvaceae. (Mallow Family.)

Malea, I.,

M. rotundifolia, L.

Sidalear, Gray.

S. malvaetlora, Gray.,

S. diploseypla, Gray,

S. vitsefolia, Gray,

Malcastrum, Gray,

M. Marrubioides,

M. Munroanum, Gray,

Fremontia, Torr.

F. Californica, Torr,

Laxatera, L.

L. assurgentiflora, Kellogg.,
Mallow.

Common Mallow,

Fields.

Meadows.

Grain fields.

Borders.

False Mallow.

Gravelly bottoms.

Hillsides.

Hillsides,

San Francisco.

\section{Linacere. (Flax Family.)}

Limum, L.,

Flax.

L. congestum, Gray,

I. Breweri, Grsy,

L. spergulinum, Gray

L. perenne, L.,

Geraniaceəe. (Geranium Family.)

Geranium, L.,

G. Carolinanum, L.,

G. crespitosum, (?) James, .

Erodum LIIter

E. cicutarium L'Her,

Lmmnanthes, R. Br.

L. rosea, Benth.,

L. Douglasii, R. Br.,

Oxalis. L.,

O. Oregana, Nutt.,

O. stricts, L.,

O. corniculata, L.,

Ptela, L.

P. trifoliata, I.,

Cranesbill.

Storksbill.

Meadows.

Alfileria.

Méadows.

Diablo.

Hillsides.

Waste places.

Sandy fields.

Meadows.

Moist meadows.

Moist meadows.

Wood Sorrel.

Yellow Wood Sorrel, . . Fields.

Fields.

Rutaceze. (Rue Family.)

Hop Tree,

Banks.

\section{Anacardiaceze. (Cashero Family.)}

Rhess, L.,

Sumach.

R. diversiloba, Torr and Gray,

Poison Oak, .

Fields.

Vitacere. (Vine Family.)

Vits, Tourn,

V. Californica, Benth.,

Thickets. 
Latin Names.

English Names.

Natural Habitat.

Frangula, Tourn. F. Californica Gray, Rhamnus, L.,

Rh. croceus, Nutt., Rh. Purshianus, Hook, Ceanothus, L.,

C. incanus, Torr. and Gray,

C. thrysoflorus (Esch.)

C. divaricatus, Nutt.,

C. rigidus," Nutt.,

C. dentatus, Torr. and Gray,

C. papillosus, Torr. and Gray,

C. crassifolus var glabratus, Gray,

C. integerrimus, Hook,

C. velutinus, Hook,

Euonymus, Tourn.

Eu. occidentalis, Nutt.,

\section{Rhamnaceae. (Buckthorn Family.)}

Alder Buckthorn.

Buckthorn.

Thickets.

Thickets.

Damp woods.

Red Root.

. . Banks,

Thickets.

Thickets.

Hillsides.

Hills.

Mountains.

Banks.

Woods.

Woods.

\section{Celastracea.. (Staff-tree Family.)}

Spindle Tree,

Moist thickets.

\section{Sapindaceae. (Soap-berry_Family.)}

Aesculus, L.,

A. Californica, Nutt Acer, L.,

A. macrophyllum, Pursh., .

A. glabrum, Torr.,

Negundo, Moench.,

N. aceroides, Moench.,

Buckeye.

California Buckeye, . . . Hillsides.

Maple.

Large-leaved Maple, . . . Banks.

Box Elder.

Mountains.

River banks.

Ficoidea.

Mesembryanthanum.

M. dimidiatum, Harv.,

Seashore.

\section{Frankeniacez.}

Frankenia, L.

F. grandifolia, Cham.,

Polygalaceze. (Milkwort Family.)

Polygala, Tourn.

P. Nutkana, Moc. \& Sessi,

Hillsides.

Leguminosae. (Pulse Family.)

Lıpmus, L.,

L. densiflorus, Benth..

L. hirsutissimus, Benth.,

L. manus, Dougl.,

- L. truncatus, Nutt.,

L. micranthus, Dougl.,

L. bicolor, Lindl.,

L. polyphyllus, Lindl.,

L. latifolius, Agardh.

L. affinis, Agardh.

\section{Lupine.}

Hillsides.

Dry places.

Fields.

Gravelly places.

Shady places.

Wet places.

Meadows. 
Latia Namk.

I. ormatus, 1)omgl.

I. allicaulus, 1),unl,

L. albitrons, bench.,

I. lithoralis. 1)omel.,

I. arborems. sims.

Trifoúum, 1...

T. eriocephalum, Nutt.,

T. Macrnoi, Hook.,

T. gracilentum, Torr. and Gray,

T. microcephalum, Pursh.,

T. variegat um, Nutt,

T. fimbriatum, Lindl.

T. heterolon, Torr. and Gray,

T. tridentatum, Lindl.

T. fucatum, Lindl.

T. amplectens, Torr. and Gray,

T. ciliolatum, Beuth.,

T. dichotomum, Hook.

T. bitidum, Gray,

T. microdon, Hook and Arn.,

T. barbigerum, Torr and Gray,

T. depauperatum, Desv.,

T. obtusifolium, Hook.,

T. spinulosum, Dougl.,

T. repens, .

Melilotus, Juss.,

11. parvifora. 1) est.

Hodronge, Lo

M. sativa, L.,

M. denticulata, Wild,

ITasackia, Dougl.

II. juncea. Benth.,

H. scoparia, Nutt.,

II. eytisoides, Benth.,

I. sericea, Benth.,

H. tomentosin, Hook and Arn.,

II. micrantha, Nutt.,

II. prostata, Nute.,

H. rigida, Benth.

11. stipularis, Benth.,

H. crassifolia, Benth.,

H. gracilis, Benth., ?

H. granditlora, Benth.,

II. maritima, Nutt.,

II. strigosa, Nutt.

H. parviflora, Benth.,

P. Purshiana. Benth.,

II. brachycaryan, Bench.

H. bicolor, Dougl.,

H. stolonifera Linull,

II. subpinnata, Tort. and Gray.,

Psorales, L.

P. physodes, Dougl.,
Evolisir Namrs.

Natiral II Abtat.

Fielile.

Sandy places.

Rocky seashore.

Large Yellow Lupine, . . Moist places,

Clover.

Dry hillsides.

Mearlown.

Roadsides.

Meaclows.

Meaduws.

Moist places.

Moist places.

IIillsides.

IIillsides.

Hillsiles.

Hillsides.

Meadows.

Meralow.

Moist places.

Hillsides.

White Clover, . . . Meadows.

Sweet Clover.

\section{Medick.}

Mearlows.

Meadows.

Fields.

Ilillsides.

Hillsides.

Hillsides.

Hillsides.

Roadsides.

Thicket.

Woods.

Moist places.

Woods.

Scashore.

Ilillsides.

Meadow.

llillmides.

River banks.

Ilillsides-

Ilillsides. 
Latin Names.

P. orbicularis, Dougl,

Thermopsis. R. Br.

Th. macrophylla, Hook and Arn., Astragalus, L.,

A. didymocarpus, Hook and Arn.,

A. tener, Gray.,

A. Breweri, Gray,

A. Douglasii, Torr. and Gray,

A. Crotalaria, Benth.

A. Menziesii, Hook.,

A. leucopsis, Torr. and Gray.,

A. curtipes, Gray.,

A. trychopodus, Nutt.,

A. oxyphysus, Gray.,

Lathyrus, I.,

L. vestitus, Nutt,

L. venosus, Muhl.

L. Torreyi, Gray,

Vicin, Tourn.,

V. gigantea, Hook.,

V. Oregana., Nutt., (truncata).

Cercis, L.,

C. occidentalis, Torr.,

Pickeringia, Nutt.

P. montana, Nutt,
A. picnostachius, Gray.

English Names.

Natural $\cdot$ Habitat.

- Thickets.

Hillsides.

Milk Vetch.

Hillsides.

Sandy flelds.

Fields.

Coast.

Coast.

Coast.

Dry hills.

Coast

Dry hillsides.

Sea shore.

Vetchling.

Thickets.

Thickets.

Vetch.

Banks

Thickets

Judas Tree.

Hillsides

Hillsides

Rosacere. (Rose Family.)

Cerasus, L.,

C. ilicifolia, Nutt.,

C. Virginiana, L.,

Nuttallia, Torr and Gray.

N. cerasiformis, Torr and Gray., Spiraea, L.,

Sp. opulifolia, L.,

Sp. ariæfolia, Smith.,

Cercocarpus, H. B. and $\mathbf{K}$.

C. parvifolius, Nutt., Acona, $\mathrm{I}$.

A. pinnatifida, Hook and Arn., Adenostoma, Hook and Arn.

A. fasciculata, Hook and Arn., Alchemilla, Tourn.,

A. arvensis, Scop.,

Cherry.

Evergreen cherry. . . . Hillsides

Choke cherry. . . . . Hillsides

Meadow Sweet.

Nine Bark.

Hillsides

Banks

Thickets

Hills and Banks

Meadows

Chemisal.

Hillsides

Lady's Mantle.

Fields

Fields

H. cuneata, Lindl.,

H. tenuiloba, Gray.

H. fusca, Lindl.,

Potentilla, L.,

$P$. anserina, $I_{\text {., }}$

P. rivalis, Nutt,

P. glandulosa, Lindl

Fragaria, Tourn.,
Cinque Foil.

Silver Weed.

Low Places

Low Places

Wet Places

Hillsides
Strawberry. 
Lativ NaMrs.

f. veses, I..,

F. chilensis, Ehrh.,

Rubrus, 'Tinurn.,

licisa. Tourn.,

12. Nuthanus, Muvino.

R. Vitifolius, Cham. \& Schlechtop

R. ursinus, Cham. \& Schlecht.

R. macropetalus, Dougl.,

R. spectalilis. Pursh.

R. occidentalis, 1.?

R. blanis, . lit.

li. gymno::arpas. Nute.

Pyrue, 1...

P. rivularis, 1) nuert.

Amedanciar. Mendie.

A. Canabensis, var, aluifolia.

Photinis, limble.,

$\mathrm{Pb}$. arbutifolia, Lindl.,

\section{Evulisu Namea.}

Bramble.

Rose.

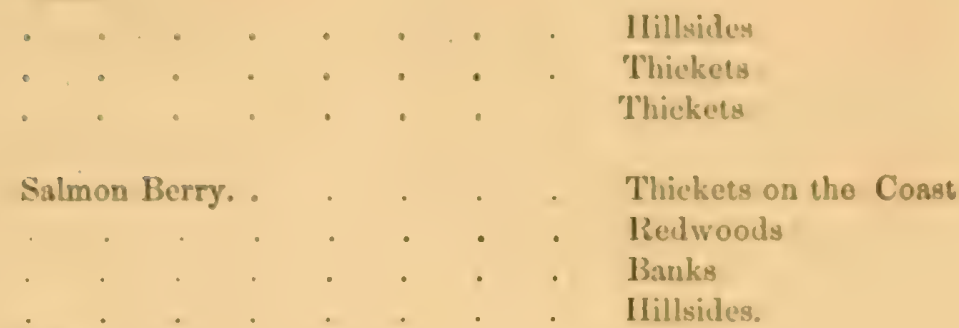

Pear.

June Berry.

Banks

Hillsides

Hillsides

Natcrar IIabitat.

'Thickets

sandy places.

Ilillsides

Thickets

Thickets on the Cosst

IIillsides.
Calycandhu, L.,

C. occidentalis, Hook.,

\section{Saxifragacexe. (Saxifrage Family,}

Thuze I-Grossulariese
Ribes, I.,
R. sanguineum, Pursh.
R. divaricatum, Dougl.
R. subrestitum, Hook and Arn.
R. speciosum, Pursh.,
R. Menziesiu, Hook,

\section{Currant Gooseberry.}

Tra: II-Mydrangiea.

Phimicipinas, L. . . . . Mock Orange or Syrinca.

Ph. Califormicus, Benth, (Lewisii Pursh.,

Thin: III-saxifragew.

Qizerifuga. I...

E. Virzina-pleis. Mirhx. var.

$S$ integrifila. Hanh.

$\therefore$ heterantha. Howk.

S. peliacia. Benth..

Tiure:ius. I...

T. unifilists. II wok.

Taüsma. Jir.

T. grandiflora, Dougl.,

T. heterophylla, Hook and Arn., (Lithophragma,)

T. affinis, Gray,

T. Bolanderi, Gray,

T. Crmbalaris, Tort. and Gray

Houdure, Lo

"

False Mitre Wort.

H. micranths, Doogl.,

11. pilosisima, Fisch and Mey.

Tolvin, Torr. and Gray,

\section{Calycanthaceze}

Sweet-scented Shrub.

Banks.

Saxifrage.

IIillsides.

Banks.

Banks.

Woods.

Woods.

IIillsides.

Rocks.

Banks.

Mountain streams.

Moist redwoods.

Ravines.

IIillsides.

Ilillsides.

Banks.

Ravines.

Alum Root.

Iavines.

Coast. 
Latin Names.

T. Menziesii, Torr, and Gray, Whipplea, Torr.

W. modesta, Torr.

Tillaea, $\mathbf{L}$.

T. leptopetala, Benth.,

Sedum, Tourn.,

S. spathulifolium, Hook.

Echeveria, D. C., (Cotyledon:)

E. lanceolata, Nutt.,
Enghish Names.

Natural Habitat.

Ravines.

Redwoods.

Crassulaceae. (Orpine Family.)

Stone Crop.

Mission.

Rocks.

Rocks.

\section{Lythraceze. (Loosc-Strife Family.)}

Iythrum, L,

L. Californicum, Torr. and Gray.,
Loose-strife.

Banks

\section{Onagraceae. (Evening Primrose Family.)}

Circaea, 'Tourn.,

C. Lutetiana, L.

Epilobium, L.,

E. angustifolium, L.,

E. tetragonum, L.,

E. minutum, Lindl.,

E. paniculatum, Nutt., (?)

Zauschneria, Presl.

Z. Californica, Presl.,

Oenothera, L.,

Oe. viminea, Dougl.,

Oe. purpurea, Curtis,

Oe. densiflora, Lindl.,

Oe. ovata, Nutt.,

Oe. graciliflora, Hook.,

Oe. viridesceus, Hook.,

Oe. contorta, Hook., .

Oe. Whitneyi, Gray..

Gayophytum, Juss.

G. strictum, Gray.,

Eucharidium, Fisch and Mey.

Eu. concinnum, Fisch and Mey.,

Hippuris, L.

$H$. vulgaris, $L$.

Callitriche, $\mathrm{L}$.

C, verna, L.,

C. marginata, Torr.,
Enchanter's Nightshade.

Willow Herb.

Evening Primrose.

\section{Haloragea.}

Mare's Tail.

Ponds.

Banks and Pools.

Banks.

Mentzelia, I.

\section{Loasacea.}

M. albicaulis, Dougl.,

M. lævicaulis, Torr. and Gray.,

Dry river beds.

Dry fields. 
Laris X.aves.

Heotisn NaMes.

Naterar IIaditat.

\section{Cucurbitacoas.}

M/gyarthis, Torr. and Gray., (Echinocystis.)

M. Oregana, Torr. and Gray.,

Big Root,

Ficlds.

\section{Umbelliferze. (Parelcy Family.)}

Hysircoot to Touru, .

Water Pennywort.

H. natans, Cyrillo,

Wet places.

H. interrupta, Muhl.,

H. Americana, $\mathbf{L}$

Wet places.

Borksia, Ruiz and Pav.,

Wet places.

B. lobata, Ruiz and Pav.,

Erymgnam, I.

E. petiolatum, Hook.,

Sanicula, S.,

S. Menziesii, L,;

S. laciniata, Hook and Arn,

S. nudicaulis, Hook and Arn.

S. bipinnatifida, Dougl.,

S. bipinnata, Hook and Arn.,

S. arctopoides, Hook and Arn.

S. tuberosa, Gray.

Deveya, Torr. and Gray.

D. arguta, Torr. and Gray.

D. Hartwegi, Gray.,

D. Kelloggii, Gray,

Ifremum, Nutt.

M. trachyspermum, Nutt.,

Apiastrum, Nutto,

A. angustifolium, Nutt.,

Cicula, L.

C. Californica, Gray.,

Sines, Lo,

S. latifolium, $\mathbf{L}$.

Carum. Ln

C. Kelloggi' Gray.,

Pimpinella, L.,

P. apiodora, Gray.,

Osmorhieso, Rafo,

O. occidentalis, Nutt,

O. nuda, Tort.

Myrrhise, Scop.

3. Bolanderi, Gray.,

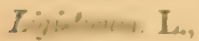

I. apifolium, Nutt,

Ange': I.

A

I'nue t.mon, I.

P. leiocarpum, Nutt.

Sanicle.

Shady places.

Dry fields.

Hillsides.

IIillsides.

Hillsides.

IIillsides.

IIillsides.

IIillsides.

Dry hillsides.

IIillsides.

Hillsides.

IIillsides.

Dry shady hillsides

Banks.

Water Hemlock.

Water Parsnip.

Wet places.

Wet places.

Caraway.

Dry hillsides.

Sweet Cicely.

Hillsides, around rockı

Shady places

Woods

Banks

Lorage.

Angelica.

Hillsides

Moigt Bankn.

IIillsirleg.

Hillsiden.

IIillsides.

Ilillsiles.

IIillaides. 
Latin Names.

P. parvifolium, Torr. and Gray.

P. foeniculaceum, Nutt.,

Heracleum, $\mathbf{L}_{\text {, }}$

H. lanatum, D. C.,

Oenanthe, $\mathrm{L}$.

Oe. sarmentosa, Nutt.

Dancus, Tourn., . . . . Carrot.

D. pusillus, Michx.,

Cancalis, L.

C. microcarpa, Hook. and Arn.
English Names.

Cow-parsnip.

Ponds.

Dry Hillsides.

Open woods.

\section{Araliaceze. (Ginseng Fam.)}

Aralia Tourn.,

A. racemosa, $\mathbf{L}$.

Spikeware.

Moist places.

\section{Cornacea. (Dogroood Fam.)}

Cornus Tourn, . . . . Dogwood.

C. Canadensis, I $\quad$ Dwarf Cornel

C. Nuttallii, And.

C. pubescens, Nutt

C. sericea I.,

Silky Cornel.

Coast.

Open woods.

Banks.

Moist places.

Caprifoliaceze. (Honeysuckle Fam.)

Lonicera, $\mathrm{L}$,

$\mathrm{L}$. involucrata,

L. Californica, Torr. \& Gray.,

Symphoricarpus, Dill.,

S. racemosus, Michx.,

S. mollis, Nutt.,

Sambucus, Tourn.,

S. pubens, Michx.,

S. glauca, Nutt.
Honeysuckle.

Bank's Twinberry.

Snow berry.

Elder.$$
\text { 年 }
$$

Moist places.

Hillsides,

\section{Banks.}

Hillsides.

Damp woods.

Hillsides.

Rubiacea. (Madder Fam.)

Galium, L.,

G. aparine, $\mathbf{L}_{\text {., }}$

G. Californicum Hook.

G. Andrewsii, Gray.

Cephalanthus, I.,.

C. occidentalis, L.,

Cleavers.

Thickets.

Button-bush.

Banks.

Valaerinaceae. (Valerian Flam.)

Plectrits.

P. congesta, Lindl,

P. macrocera, Torr. and Gray., .

Hillsides.

Hillsides. 
Compositze. (Composile Family.)

\section{Tubuliflorae.}

Bulbestylis, 1)C.,

B. Californiea, Gray.

Nardoemia, Cap.,

Sweet Coltsfoot.

N. palmata, llook. Ademoramion, Ilocks.,

A. bicolor, Hook.,

Corethrogyne, DC.,

C. incans, Nutt.,

C. filaginifolia, Nutt.

C. tomentells, Gray.,

C. spacthulata, Gray.

Swricoaarpm, Nocs,

S. rigidus, Lindl.,

Aster, $\mathrm{L}$.

.

\section{Dry Creekbeda.}

Muist Banks.

Redwoods.

Dry Hills.

Dry Hills.

Dry Hills.

Seashore.

White-topped Aster.

\section{Dry Banks.}

Aster.

A. Durandi, Nutt.,

A. laxifolius, Torr. \& Gray.? Frigeron, L.,

E. Canadense, L.

F. supplex, Gray.,

E. glaucum, Ker.

E. glalvellum, Nutt.

Gukierroziç, Lag.,

G. Californica, Gray Solidayo, Le,

S. spiciformis, Gray.,

S. Californica, Nutt,

S. elongata, Nutt.

S. occidentalis, Nutt.,

Linaryris, Lobel.,

I. arborescens, Gray., Aplopoppu, Cass.

A. linearifolius, DC.,

A. microphylla, Nutto,

Grindelia, Wilid.,

G. hirsutula, Hook.

G. robusta, Nutt.,

Pendachede, Nutt.,

P. aurea, Nuth.

Chrymopsi, Nute.

Cb. Bolanderi, Gray.

F

Fleabane.

Horseweed. . . Fields.

Moist Banks.

Hillsides.

Coast.

'Fields.

Fields.

Golden Rod.

Hills.

Open Woods.

Hillsider.

Moist Places.

Moist Banks.

Redwoods.

Monte de Diablo.

Sandy Fields.

Dry IIillsides.

Seasbore.

IIillsides.

Golden Aster.

Dry Hillsides.

Seashore.

A pimedeatr, Gray.

A exilis riray.,

Becchoris, In,

13. eonsanguirses ITC.

Groundsel Tree.

IIillsides.

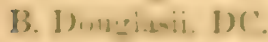

13. siminua. I) '.,

Plucher, Casa,

Marsb Fleabane.

IIillsides.

Mnist I'laces.

Riverbeds.

P. camphorata, I)C.

Salt Marnhea. 
LATIN NAMIES.

Micropus, L.,

M. Californicus, Fisch \& Mey.,

Psilocarphes, Nutt.,

P. tenellus, Nutt.,

P. globiferus, Nutto,

Stylocline, Nutt.,

St. gnaphaloides, Nutt.,

Evax.

E. caulescens, Benth.,

Blennosperma, Less.,

B. Californicum, Torr. \& Gray.,

Franseria, Cav.,

F. Chamissonis, Less,

F. bipinnatifida, Nutt.,

F. Hookeriana, Nutt.,

Xanthium, Tourn.

X. strumarium, Is,

X. spinosum, $I_{\text {s, }}$

Wyethia, Nutt.

W. helenioides, Nutt.

W. angustifolia, Nutt.,

W. glabra, Gray.,

Balsamorhiza, Hook.

B. macrophylla, Nutt.,

B. deltoidea, Nutt.,

Helianthus, $\mathbf{L}$.,

H. Californicus, DC.,

H. Bolanderi, Gray.,

H. exilis, Gray.

Helianthella.

H. Californica, Gray.,

Coreopsis, $\mathrm{L}_{\text {., }}$

C. calliopsidea, DC., ?

Bidens, L. Bur.,

B. chrysanthemoides,

Leptosyne, DC.

I. maritima, Gray.,

L. Douglasii, DC.,

Pugiopappus, Gray.

P. Bigelovii, Gray.,

Chaenaetrs, DC.

Ch. heterocarpha, Gray., .

Ch. tanacetifolia, Gray.,

Ch. lanosa, DC.,

Bahia, Lag.

B. artemisiæfolia, Lep.,

B. confertiflora, DC.,

B. lanata, Nutt.,

B. arachnoidea, Fisch \& Mey.,

Lasthenra, Cass.

L. glaberrima, DC.,

L. glabrata, Lindl.,
English Names.

Natural Habitat.

Hillsides.

Hillsides.

Hillsides.

Dry Hillsides.

Hillsides.

Moist Hillsides and Meadows.

Sandy Seashore.

Sandy Seashore.

Sandy Seashore.

Cookle bur.

Common Cockle bur.

Fields.

Spiny Cockle bur.

Waste places.

Hillsides.

Meadows.

Hillsides.

Hills.

Hillsides.

Sunflower.

Banks.

Hillsides.

Meadows.

Hillsides.

Tickseed.

Marigold.

Michx.,

Moist Hillsides.

Swampy Places.

Salt Marshes.

Sandy Hills.

Sandy Hillsides.

Dry Hillsides.

Chaparral.

Sandy Hills.

Seashore.

Dry Hillsides.

Coast.

Thickets.

Wet Places.

Wet Places. 
Berrielia, 1)C.

B. chrysestoma, (Fray., (also var macrantha)

13. maritima, liray.

13. tenervima, I)

Dichesels, Vute.

1). uligin ssa, Nutt.

R. promsprias,

R. lefrocialus, liray.,

HL:mierm, 1.

11. paberulum, I) ('.,

II. Iiolamderi, Ciray..

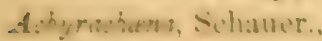

A. mollis shomer.

Layia, Hook \& Arn.

I. hiersoioides, Hook \& Arn.,

I. carnosa, Nutt.,

I. gaillardioides, Hook \& Arn.,

I. heterotricha, Hook \& Arn.,

L. plstyglossa, Gray.,

I. calliglossa, Gray.,

L. chrysanthemoides, Gray.,

Hemizonia, DC.

H. corymbosa, Torr. \& Gray.

H. angustifolis, DC.

H. pungens, Torr. \& Gray.,

11. virgata, Gray.,

H. luzulsefolia, DC.,

11. macratenia. 1)(?

1I. milis, Bantil.,

Cisigundiontio, UC.

C. truncata, DC.

C. cephalotes, DC.

\section{Y... Nitit.}

I. r.ou ciana, Niti,

Me:r:m I)(.

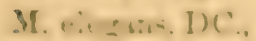

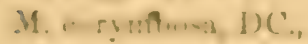

Ma: ... M .in:a..

M. sativa, Molins var congesta,

M. racemosa, Torr. \& Gray.,

M. dissilifiora, Tort. \& Gray.

Borpecorpus, Nutt.

II. madarioides, Nutt.

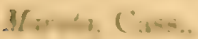

M. ( a vila, DC.

A.? $\cdots, I$.

A. Mill-inlium, I.

MC: $:$ DC.

M. Iancentatas var in.jor,

M. mimer, I) '.

Comegrone. 1.ent.,

C. сзтила, Less,

Matricaria, I.. sneczo Treed.

Hills.

Seashoro.

Sulinas Pluins.

Mesulows.

Dry IIIlsides.

Wet Places.

Coast Swampy Placea.

Ficlds.

IIillsides.

Seacoast.

Sandy IIills.

Hillsides.

IIillsides.

Hillsides.

IIillsiles,

Hillsides.

Clay Soil.

Dry Valleys.

Hillsides.

Iillsides.

San Jose Valley.

Dry IIils.

Dry Hills.

Sandy Fields.

Ficlds.

Fields.

Fields.

Ficlis.

Ficlds.

May-weed.

Dry IIillsidon.

Iondsides.

latrow.

Ficlda.

Moist Meadows.

IIillm.des.

Wild Chamomile.

Salt Marshew. 


\section{Latin Names.}

M. discoidea, DC.,

Cotula, Gærtu.,

C. coronopifolia, L

Tanacetum, L.,

T. Huronense, Nutt., Artemisia, L.,

A. pyonocephala, DC.

A. dracunculoides, Parsh.

A. Canadensis, Michx.,

A. filifolia, Torr.,

A. discolor, Dougl.,

A. vulgaris, $\mathbf{L}$.,

A. pachystachya, DC.,

A. Californica, Less.,

Soliva, Ruiz \& Pav.

S. daucifolia, Nutt.,

Gnaphalium, L.,

G. Californicum. DC., var rubrum,

G. palustre, Nutt.,

G. purpureum, L.,

G. ramosissima var erubescens,

G. Sprengelii, Hook \& Arn.,

Antenniaria, Gaertn.,

A. margaritacea, $\mathbf{R}$. Br.,

Filago, Tourn.,

F. parvula, Torr \& Gray,

Cacalia, $\mathbf{L}$.

C. Nardosmia, Gray.

Senecio, L.,

S. vulgaris, $\mathbf{L}$.,

S. Douglasii, DC.,

S. aronicoides, DC.,

S. Bolanderi, Gray.,

S. Mendocinensis, Gray.,

Arnica, L.,

A. parviflora, Gray.,

A. cordifolia, Hook. var. discoidea

Lessingia, Cham.,

L. ramolosa, Gray.,

L. virgata, Gray.,

L. Germanorum, Cham.

L. leptoclada, Gray,

Centaurea, I.,

C. Melitensis, $\mathbf{L}$.,

C. solstitialis, $\mathrm{L}$.,

Cirsium, Tourn.,

C. undulatum, Spreng.,

C. Coulteri, Harv. \& Gray.

C. acaule, All.,

C. Californicum, Gray.

Silybum, Vaill.,

S. Marianum, Gærtn.,
English Names.

Natural Habitax.

Waste Places.

Salt Marshes.

Tansy.

Wormwood.

\section{Seashore Cliff House.}

Sandhills.

Banks.

Fields.

Hillsides.

Fields.

Fields.

Fields.

Sandy Fields.

Cudweed.

Meadows.

Hills.

Wet Places.

Hillsides.

Thickets.

Seashore.

Everlasting.

Cotton Rose.

Indian Plantain.

Groundsel.

Arnica.

Thickets.

Fields.

Open Woods.

Waste Places.

Dry Beds and Fields.

Hillsides.

Rocks, Seashore.

Meadows.

Dry Hillsides.

Thickets.

Waste Fields.

Dry Fields.

Dry Fields.

Sandy Fields.

Star-Thistle.

Fields.

Grain Fielda

Thistle.

Hillsides.

Sandy Fields.

Dry Hillsides.

Fields. 
Cialsis. 1)C..

C. linearifilia, 1) ('..

C. macruchata. Gray.

C. Douglasii. I)(..

C. Bienelovii, liray.

C. aphantenespha, liray,

c. cyelecarpha liray,

C. Bulanderi, (iray.

C. enetla liray.

C. laciniata. Gray.

Siephomemerit. Sitt..

St. panienulata. Xutc.

st. virgata. liray.

Retines gair. Nute.

R. Califurnita. Xute.

Hienaciva, 'Tourn.,

H. albiflorum, Hook,

H. triste, Willd.

Ionteotina I)

M. Californica, DC.

M. obtusa, Benth.,

M. incana, Gray,

M. parviflora, Bentb.

Crepis, Lo,

C. occidentalis, Nutt,

Malacolepis, Harv. \& Gray,

M. Coulteri, Harv. \& Gray,

Mecrorhynchus, Lesis.

M. grandiflorus, Torr. \& Gray,

M. heterophyllus, Nutt.

M. bumilis: Benth.,

M. retrorsus, Benth.

M. elatus, Nutt.,

Soncher, $\mathrm{L}$.

S. asper, Vill,

8. oleraceus, $\mathbf{L}$

Dypochaerio, glabra, L., (introduced)

\section{Ligulinorae.}

Hawkweed.

Sow Thistle.

Spring-leaved Sow Thistle,

Common Sow Thistle.
Gravelly Hillsides.

Ficlds.

Meadows.

Meadow.

Around Bushes.

Around Bushes.

Grainticlels and Meadows.

Fichls.

Meadows,

Hillsiles.

Hillsides.

Gravelly Hillsicles.

Woods.

Woods。

Fields.

Hillsides.

Hillsides,

Hillsides.

Dry Hillsides.

Sandy Hills.

Meadows.

Fields.

Fields.

Open Woods.

Fields.

Fields.

Fields.

San Francisco.

Donowingie, Torr.

Lobeliacere. (Lobelia Fiamily.)

D. elegane, Torr. .

Borders of Swamps.

Campanulaceae. (Campanula Family.)

Cempomils, Tourno,

Bellflower.

C. linnseifolia, Gray,

C. prenanthoides, Gray,

Heterocodom. Nute?

H. rariflorum, $\mathbf{N}$ utt,

Githopeis, Nutt.,

G. opecularioides, Nur.,

Swampy Places.

Redwoods.

Grassy Plains.

Hillsides. 
LATIN NAMES.

Vaccinium, L.,

V. avatum., Pursh.,

V. parvifolium, Smith, Arbutus, Tourn.,

A. Menziesii, Pursh., Arctostaphylos, Adams.,

A. tomentosa, Dougl.

A. glauca, Lindl.,

A. pungens, H. B. \& K.

A. nummularia, Gray,

Gaultheria, Kalm.,

G. Shallon, Pursh.,

Asalea, L.,

A. occidentalis, Torr. \& Gray,

Rhododendron, $\mathrm{L}$.

Rh. Californicum, Gray,

Ledurm, $\mathbf{L}$.

L. glandulosum Nutt.,

Pyrola, Tourn.

P. rotundifolia, L.,

Chimaphila. Pursh.,

Ch. umbellata, Nutt.

Allotropa, Torr. \& Gray,

A. virgata, Torr. \& Gray,

Plantago, L.,

P. maritima, L.,

P. Bigelovii, Gray,

P. Patagonica, Jacq.

Armeria, Willd.

A. vulgaris, Willd. var.

Statice, Tourn.

St. Limonium, L., .

Trientalis, $\mathrm{I}_{\mathrm{L} \text {, }}$

T. Europaa, L.

Glaux, L.,

G. maritima, L., Anagallis, Tourn.,

A. arvensis, $\mathrm{L}_{\text {., }}$ Dodecatheon, I.,

D. Meadia, L.

Phelipcea, Tourn., .

Ph. uniflora, Gray.t

Ph. fasciculata, Spreng.,
Enguish Names.

Natural Habitat.

Ericaceae. (Heath Family.)

Blue berry.

. . . . . Hillsides.

Madrona.

. . . Redwoods.

Bearberry. . . . Manzanita.

Hillsides.

Gravelly Plains \& Hills.

Hillsides.

Gravelly Plains.

Wintergreen.

False Honeysuckle.

Redwoods.

Redwoods.

Rose Bay.

Labrador Tea.

Shin Leaf.

Pipsissiwa.

Redwoods.

Coast, Swampy Places.

Thickets on the Coast.

Thickets on Hills.

Open Woods.

Plantaginaceae. (Plaintain Family.)

Plantain.

Seashore.

Hillsides.

Plumbaginaceae. (Leadroort Family.)

Marsh Rosemary.

Sand Seashore-Hills.

Salt Marshes,

\section{Primulaceae. (Primrose Family.)}

Chickweed Wintergreen.

Sea Milkwort.

Common Pimpernel.

Cowslip.

Thickets.

Seashore.

Pimpernel.

Fields.

Hillsides aud Meadows.

Orobanchaceae, (Broom-Rape Family.)

Broom-Rape.

Moist Hillsides.

Hillsides. 
Lerix Pans.
Evousm Names.

Nitikal. Hamitat.

\section{Scrophulariaceze. (Figroort Fanuily.)}

I:mase, Tourn..

L. Comblensis spren:.

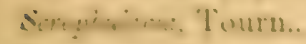

S. nudisa. I...

T.M. . .

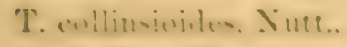
$A=1$.

A. El:anduL-um, limil..

A. lireweri, lima:

A. vayation liray,

A. vagans, var., Bolanderi, Gray,

Collimair. Nutt.,

C. Haw - $\therefore$ is lisuth..

C. parviflora, Dougl.,

C. bicolor, Benth.,

C. riolaces, Nutt..

C. grandiflora, Dough,

C. corymbosa, Ilortu。,

C. solitaria, Kellogg,

Pentetemon. Mitch.,

P. Menziesii, Hook.,

P. azurius, Benth., var.,

P. centranthifolius, Benth.,

molyes Jarey.

W. antionais, 1morl..

D. Len. Xint.

1). Gatin w... Xinu.

if 1 . 1 .

II. Lueen., L.,

M. cardinalis, Dougl.

M. mosechatus, Dongl.,

M. inconspicuous., Gray.

Eunamus. Bench.

E. Douglasii. Benth.,

E. Coulteri, Benth..

Veronica, L.

V. Arnericana. Schn,

Cordylunethus. Nutt.

C. pilosus, (iray.

C. maritimus, Nutt.

Castilleia. Mutis.,

C. parvithora, Bong.,

C. affinis. Ilook \& Arn.,

C. Latifolin, Hook ot Arz.,

C. foliolosa. Hook. \& Arna .

Grilestpue Nan.

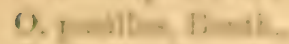

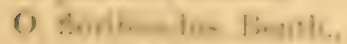

o. erwe.:- Finent.

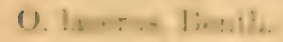

Toal-Flax.

Figwort.

Suapdragon

Shady IIillsides.

IIillsides.

Banks.

Banks.

Redwoods.

Collinsia.

Thickets.

Fields.

Hillsides.

IIillsiles.

Moist Places.

Sandy Seashore.

Fields.

Beard Tongue.

Rocks.

Sandy Banks.

Sandy Hills.

Mossy Shady Banks.

IIillsides.

Monkey Flower.

Springy Places.

Borlers of Streams.

Moist Places.

Moist Places.

IIillsidles.

Irillsides.

speedwell.

Springy Places.

Ilillsisles.

Salt Marsh.

I'ainted Cup.

Hiillsiles.

Hillsiles.

C'onet.

Dry IIillsiden.

Mealow.

Moist Mealows.

Sandy Fields.

Mnist Burden. 


\section{LATIN NAMES.}

o. lithospermoides, Benth., .

O. faucibarbatus, Gray,

O. densiflorus, Benth.,

O. castillejoides, Benth.

o. attenuatus, Gray,

O. purpurescens, Benth.,

Pedicularis, Tourn,

P. densiflora, Benth.

P. attenuata, Benth.,

\section{English Names.}

Naterat Habitat.

Moist Fields.

Moist Fields.

Moist Meadows.

Moist Meadows.

Moist Meadows.

Moist Meadows.

Lousewort.

Shady Hillsides.

Shady Hillsides.

Verbena, L.,

V. prostata, R. Br.

V. hastata, L.,

Verbenacez. (Vervain Family.)

Vervain.

Fields.

Water Courses.

Labiatæ. (Mint Family.)

Mentha, L.

M. Canadensis, L.,

Lycopus, L.,

Europæus, L,

Pogogyne, Benth.

P. Douglasii, Benth.,

P. serpylloides, Gray,

Salvia, L.,

S. carduacea, Benth., Audibertia, Benth.

Mint.

A. grandiflora, Benth., .

A. stachyoides, Benth.,

Lophanthus, Benth.,

L. anisatus, Benth.,

Brunella, 'Tourn.,

B. vulgaris, L.,

Scutellaria, L.,

S. tuberosa, Benth.,

S. Bolanderi, Benth.,

Monardella, Benth.

M. villosa, Benth.,

M. odoratissima, Benth.,

M. Breweri, Gray,

Marrubium, L.,

M. vulgare, L.,

Stachys, L.,

St. ajugoides, Benth.,

St. Chamissonis, Benth.,

St. Nuttallii, Shuttlew,

Micromeria, Benth.,

M. Douglasii, Benth.,

Trichostema, L.

T. lanceolatum, Benth.,

T. lanatum, Benth.,

T. laxum, Gray,

Sphacele, Benth.,

S. calycina, Benth.,
Wild Mint.

Water Horehound.

Sage.

Springy Places.

Borders of Streams.

Dry Meadows.

Dry Places.

Dry Hillsides.

Dry Hillsides.

Thickets.

\section{Giant Hyssop.}

Self-Heal.

Skull-Cap.

Horehound.

Common Horehound.

Hedge-Nettle.

Yerba Buena.

Blue Curls.

Sandy Banks.

Hills near the Coast.

Hillsides.

Hillsides.

Dry Rocky Banks.

Dry Rocky Banks.

Hillsides.

Fields.

Thickets.

Moist Borders.

Hillsides.

Sandy Soil.

Springy Places.

Hillsides.

Around Springs.

Dry. Soil. 
Boraginaceae. (Borayo Fumily.)

Lithospermum, 'T'uurn., I. hirsutum, Mey. Amsiniver. I.ehmo..

A. intermetia, Fiscli \& Mey. A. spectabulis. Fiseh of Mey. Eribrichum, Sichral.,

E. ('alifornicum, 1)(..

E. fulvum, 1)

E. ('horisimum, 1)'.

E. plebejum, 1$)$..

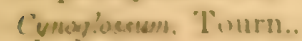
C. gramile. 1)omel. Heniveropum. Tourn.,

II. Curassavicum, L..

Eriadyction, Benth.,

E. Glutintsum, Benth..

Rumumatin, C'ham.

R. siculensis, cham.,

Bemepticia. Sinu.

X. yarvifhra. Benth.

X. atemaria Fiseh et Mey.

X. insignis, Bench..

X. penlunenlat:a, benth.,

D. aurita Lim Il..

E....sics. I.

E. chrysanthemifolia, Benth., Phacelia, Juss.

Ph. circinata, Jacq,

Ph. ramosissima, Dougl.,

Ph. tanacetifolia, Benth.,

Ph. malvaefolia, Chamo,

I'b. ciliata, Benth.,

Ph. divaricata, Benth.,

Ph. loaverfulia, Benth.,

Ih. Donglasii. Benth.,

Einmemanthe, Benth.

E. penduliflora, Benth.,

Callowis, Sintt.

C. grandiftora, Dougl.,

C. gracilis, Dougl.

C. glutinnsa, J3enth.,

Severretie, Kuiz \& Yav.

X. leucocephala, Benth.

9. equarrosa llook \& Aro.

N. atratyloides. Honk \& Arn.
Puccoon.

Fields.

Hillaider.

IIillsides.

Meadows.

Meadows.

Meadows.

Meadows.

Hound's Tongue.

Heliotrope.

Hillsides.

Alkaline Soil.

Dry Hillsides.

Moist Shady Placos.

Hillsides.

Thickets.

Fields.

Hillsides.

Thickets.

Hillsides.

IIillsiles.

IIillsides.

Waste Places.

Shady Places.

Hillsides.

Hillsides.

Hillsidles.

IIillsudes.

Millsidew.

\section{Polemoniacea.}

Wonils.

Hillsides.

Hillsilles.

Mrist Placen.

Hillminleg.

Hillsiden. 
LATIN NaMes,

Gilia, Ruiz \& Pav.

G. densiflora, DC.,

G. elongata, Steud.,

G. virgata, Steud.,

G. capitata, Dougl.,

G. multicaulis, Benth.

G. tricolor, Benth., .

G. arenaria, Benth., .

G. dichotoma, DC., .

G. pharmaceoides, Benth.

G. micrantha, Steud.,

G. Intea, Steud.,

G. androsacea, Steud.,

G. grandiflora, Steud.,

G. ciliata, Benth.,

Polemonium, Tourn.,

P. coruleum, I.,
English Names.

Natural Habitat.

Hillsides.

Hillsides.

Hillsides.

Sandy Fields.

Hillsides.

Open Wood \& Meadow.

Sandy Fields.

Hillsides.

Hillsides.

Hillsides.

Hillsides.

Sandy Fields.

Hillsides.

Hillsides.

Greek Valerian.

Banks.
Centianaceze. (Gentian Fam.)

Erythraa, Pers.,

E. Muhlenbergii, Griesb.,

Gentiana, L.,

G. crocea, Engelm.

G. calycosa, Griesb., (?)

Menyanthes, L.

M. trifoliata, L.,

\section{Centaury.}

\section{Gentian}

Moist Meadows.

Moist Meadows.

Moist Places.

Swamps.

Convolvulaceae. (Convolvulus Fam.)

Convolvulus, $\mathrm{L}$.,

C. arvensis, L.

C. Californicus, Choisy.,

Calystegia, B. Br.

C. Soldanella, R. Br.

C. sepium, R. Br.

Ipomea, $\mathbf{L}$.

S. sagittata, Desf.,

Cuscuta, Tourn.,

C. Californica, Choisy.,

C. subinclusa, Duran \& Hilg.,

Solanum, Tourn.

Bindweed.

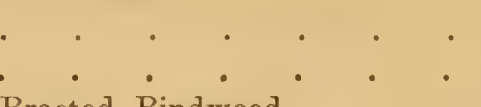

Fields.

Banks and Fields.

Bracted Bindweed.

Dodder.

Hillsides.

Seashore.

IIillsides.

On Phacelia.

Salt Marshes.

Solanaceze. (Nightshade.)

S. umbelliferum, Esch.,

S. nigrum, L.,

Nicotiana, L.

N. quadrivalvis, Pursh.,

Common Nightshade,

Thickets.

Waste Places.

Tobacco.

Fields.

Apocynaceae. (Dogbane Fam.)

Apocynum, Tourn.,

A. androsamifolium, L.

Indian Hemp.

Dogbane,

Indian Hemp.
Hillsides.

River Banks. 
Asclepiadaceae. (Milluned Fum.)

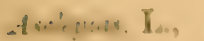

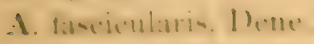

A. eriocarys, Benth., A. - rever, Eill.

A. cordifolia, Benth.,

A. tomentosa. Torr.

F. ..imes, 'Tourn.,

F. (Tregana. Nutt.

Arietolorhin. Tourn.,

A. Californica, Gray, A rammo, Tourn.

A. Hookeri. Field.
Milkweed.

Ilillnides.

Hillsides.

Green Milkweed.

Ilillnirles.

Ilillsides.

Oleacere. (ONine Fim.)

Asb.

liver l3anks.

Aristolochiaceae. (Birtherort Fumily.)

Wild Ginger.

Thickets.

Damp Places.

Chenopodiacere. (Goorfoot Family.)

Cheneportions, I. .

Ch. allum, L.

Ch. urbicum, L.

$\mathrm{Cb}$. anthelminticum. I.

Obiome, Gartn,

Goosefoot.

Pigweed.

Wormseed.

Waste Places.

Waste Places.

Waste Places.

Glasswort.

Salncomia. Tourn.

- I.ertianca L..

\section{1. .... Ti, Trm.}
A. 1...m 1 .
A rietrotle lin. I.

$.1 \cdot \cdot 3, \ldots$

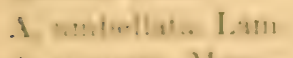

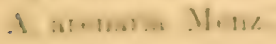

Amarantaceae. (Amuranth Fimily.)

Fields.

Fields.

Nyctaginaceae. (Fonr o'clock Family.)

Sandy Scaslore.

sandy Seashore.

Polygoniacere. (Buckecheat Fumily.)

Palygomano, I.

Knotweed.

P. aviculare, $\mathbf{L}$, .

Doorweerl.

P. maritinum. I.

P. Paronvehis, Cham.,

P. acre. H. B. \& K.,

himen I.

It mortimes I.

Ii. $1, \cdot \cdots+\ldots \mid, 1$..

1 imonen. I: lir

(1. an-mantalla. Nuts.

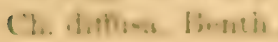

\section{Salt Marshes.}


Latin Names.

Ch. pungens, Benth.,

Ch. nudicaulis, Nutt.

Pterostegia, Fisch \& Mey.

P. drymarioides, Fisch.\& Mey.,

Eriogonum, Michx.

E. ciliatum, Torr.,

E. latifolium, Smith.,

E. parvifolium, Sm.,

E. leucocladon, Benth.,

E. compositum, Dougl.,

E. orthocladon, Torr.,

E. umbellatum, Torr,

E. polifolium, Benth.,

E. virgatum, Benth.,

E. acetoselloides, Torr.,

E. rotundifolium, Benth.,

Oreodaphne, Nees.

O. Californica, Nees.,

Laurel.

Thymelaceze. (Mezereum Fam.)

Dirca, L.,

D. palustris, $\mathrm{L}$.

Leatherwood.

Loranthacea. (Mistletoe Family.)

Phoradendron, Nutt.,

$\mathrm{Ph}$. flavescens, Nutt., var. pubescens Englem.,

False Mistletoe.

Arceuthobium, Bieberst.

A. campylopodium, Englem.,

Saururaceze. (Lizard's Tail Fumily.)

Anemiopsis, Hook.,

A. Californica, Nutt.

Tricerastes, Presl.

T. glomerata, Presl.,

Datiscacez.

Euphorbiaceae. (Spurge Family.)

Euphorbia, L.,

E. leptocera, Englem.,

E. crenulata, Englem.

E. Lathyris, L.,

Croton, L.

C. procumbens, Esch.,

Eremocarpus, Bunge.,

E. setigerus, Benth.

Simmondsia, Nutt.

S. Californica, Nutt,
Spurge.

(Naturalized.)
Naturat Habitat.

Sandy Fields.

Sandy Fields.

Hillsides.

Hillsides.

Sea Coast.

Hillsides.

Hillsides.

Sea Coast.

Dry Hillsides.

Rocks.

Sandy Hills.

Grassy Places.

Grassy Places.

Rocky Ravines.

Streams \& Hillsides.

Hillsides.

On Oaks.

On Pine Trees.

Pools.

Fields.

Hillsides.

Monterey.

Sandy Fields.

Dry Valleys.

Hillsides. 


\section{Urticacoae. 1.1 !., lim}

(i, . Ti, : : th.

l. $\ldots, \cdots, 1 \ldots$

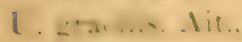

\section{li:ms=11...1.}

(i. , ill:00.9

(i) I rew und, 1, ir:ay.

(i) limatim lims.

Platanacene. (Plone Tren Fhm.)

I $\cdots \cdots \cdot \mathbf{L}$

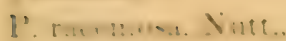

Juglandacere. (Walnut Fam.)

I. $\cdot$. I

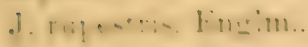

$(\because-\cdots 1$

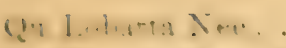

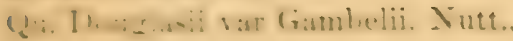

Qu. Agrifolia Nee

Qu. chrvsolepis, Liebm.

Qu. Sonomensis, Benth.

Qw. Wislizeni, Engelm.

Qu. densiffora Hook.,

Ceulenes, Tourn.,

C. chrysophylla, Dougl.

Corylus, Tourna

C. rostrata Aic.
Buttonwood, Sycamore.
Walnut.

Walnut Creek. llillsilles.

Hillsides.

Swamps.
Banks.

Waste Grounds.

Thiekets.

\section{Carryaceae.}

\section{Cupuliferae. (Oak Fam.)}

Oak.

\section{Deciduous. (Fruit Annual.)}

White Oak, Burr Oak,

White Oak.,

Valleys.

Gravelly Soil.

Evergreens. (Finit Annual.)

Common Live Oak., Mountain Live Oak.

Deriduous. (Fruit Bienninl.)

Black Oak,

Erergreen. (Fruil Bienial,)

Live Oak,

Chestnut Oak.

The Ahrubby ()alkn are ornitted.

Chestrout. Chinquapio.

Harelnut.

Beakerl Hazelnut. Hillwisles.
Hillsides.

Valleys.

Hills near Const.

Northern Valleys.

liedwools.

Myricaceae. (Sieed (inle Fam.)

Myrice. Lo

M. Californica, Cham, \& Scbl.
Baybery, Wax-Myrtle.

Moint Banka. 
Latin Names.
English Nayes.

Naturat Habitat.

\section{Betulacex., (Birch Fam.)}

Almus, Tourn,

A. viridis, DC.
Alder.

Green Alder, .

Water Courses.

Salicacere. (Willow Fam.)

Salix, Tourn.,

S. lucida, Muhl., (lasiandra Benth)

S. longifolia, Muhl.,

S. Hindsiana, Benth, (S. sessilifolis, Nutt.,)

S. Bigelovii, Torr.

S. lasiolepsis, Benth.

S. Coulteri, Aud.,

S. capreoides, Aud.,

S. l rachystachys, Benth.,

Populus, Tourn.,

P. monilifera, Ait.

\section{Willow.}

Willow.
Bolinas Bay.

Streams.

Swamps and Banks.

San Francisco.

Streams.

Moist Places.

Moist Places.

Open Woods.

Streams.

\section{Coniferae. (Pine Fam.)}

Pinus, Tourn.,

Leaves two in a close sheath; cones persistent.

P. muricata, Don., .

Bishop Pine,

Hills on the Coast.

P. contorta, Dougl. (P. Bolanderi Pal ?)

Twisted Pine, .

Coast.

Leaves three in a close sheath; cones persistent.

P. insignis, Dougl.,

P. tuberculata, Don.,

P. Sabiniana. Dougl.,

P. Coulteri, Don.,

Monterey Pine,

Sea Coast.

Hillsides.

Dry Hillsides.

Hillsides.

Leaves three in a close sheath; cones falling after shedding the seed.

P. ponderosa, Dougl.,

Yellow Pine,

Higher Hills.

Leaves five in a close sheath: cones falling atter shedding the seed.

P. Lambertiana, Dougl.,

Picea, I.,

P. grandis, Dougl.,

Albies, $\mathrm{L}$.,

A. Mertensiana, Bong.,

A. Douglasii, Lindl.,

A. Menziesii, Dougl.,

Thuja, Tourn.,

Th. gigantea, Nutt.

Libocedrus, Endl.,

L. decurrens, Torr.,

Sequoia, Endl.,

S. sempervirens, Endl.,

Torreya, Arnott,

T. Californica, Torr
Sugar Pine,

Fir.

White Fir.,

Hemlock \& Spruce.

Hemlock Spruce,

Spruce,.

Black Spruce, .

Arbor Vitæ.

Bastard Cedar.

Redwood.

Nutmeg.
Highest Hills.

Coast Range.

Northern Coast Range.

Redwoods.

Northern Redwoods.

Northern Coast Range.

Eastern Coast Range.

Coast Range.

Redwoods. 
L.om Nines.

Cinmereser, Tourn.,

C. m.., rocarpa,

C. Macnabiana, Murrey.
k.NaLisu NAMEs.

Natinal IIAmtat.

\section{A spectes of shrubby juniperus oceura iparingly on Monte del Dlublo; th mpect do namo tu unknown uo me.

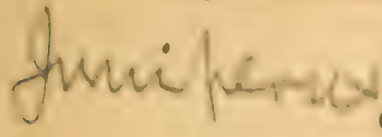 \\ CLASS II.-MONOCOTYLEDONOUS PLANTS.}

Araceae. (Arum Fum.)

Symplonarpme, Sabisb.,

S. Kamtschaticus, Bong.

Loma, I.

1.. tri-nia 1.

Typha. Tourn.

T fatim in 1.

Potamogetom, Tourne,

P. natane, $\mathbf{L}$.

I'. pectinat us, $\mathrm{L}_{\text {., }}$

Heleroslylus, Hook.

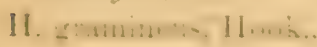

Triglochin. L.

T. maritimum, L.,

Alimma, L..

A. Plantago, Lo, var Americanum,

A. Californica, Torr.,

Mabenoris. Willd.

II How os Lindle.

II +1. - in- Iin 11 .

Surm.: Ra Rari.

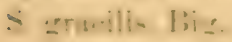

$\therefore$ H....

E....... Hall

E. Am-riman Indi

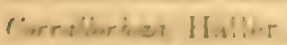

C. mriana Lin. II.

Cypr.pedium. I.

\section{Cypress.}

Monterey Cypress, . . . Coast.

Dry Hillsides.
Skunk Cabbage.

Lemnacese. (Duekroced Fram.)

Duck's Meat.

Typhaceae, '(Cat Tuil Fim.)

Cat Tail Flag.

Naiadacere, (Ponduced F(am.)

Fwamp.

Siturnamt Watur.

Swamps on the Coust.
Ponds.

Salt Water Marshes and Streams.

M:ar-hy l'lases.

Alismaceae, (Water Plantain Fam.)

Arrow Grass.

Water Plantain.
Salt Marshes.

Swampy Places.

Swampy Places.

Orchidacese, (Orchis Fum.)

Rein Orchis.

Ladies Tresses.

Swamps.

Swamps.

IIillsides.

Redwoods.

Coral Root.

Borders of Streams.

Lady's Slipper.

Words. 
Latin NaMes.

English Names.

Natural Habitat.

C. Californicum, Gray.,

C. sp. ?

Swamps.

Redwoods.

I ridacea, (Iris Fam.)

Iris, L.,

I. longipetala, Herb., .

I. macrosiphon, Torr \& Gray.,

I. Douglasiana, Herb.,

Sisyrinchium, L.,

S. Bermudiana, L.

S. lineatum, Torr.,
Flower-de-Iuce.

$\begin{array}{lll}\cdot & \cdot & \cdot \\ \cdot & \cdot & \cdot \\ \text { Blue-eyed Grass. }\end{array}$

\section{Liliacea, (Lily Fam.)}

Trillium, L.,

T. sessile, $L_{\text {, }}$

T. ovatum, Pursh.,

Veratrum, Tourn.,

V. fimbriatum, Gray,

V. Californicum, Durand,

Xerophyllum, Michx.

X. setifolium, Michx.,

Tofieldia, Huds.,

T. glutinosa, Willd.,

Prosartes, Don.

P. Hookeri, Torr.,

P. trachyandra, Torr.,

Streptopus, Michx.,

St. amplexifolius, DC.,

Antuclea, Kth.

A. Fremonti, Torr.,

Scoliopus, Torr.

S. Bigelovii, Torr.,

Clintonia, Raf.

C. Andrewsiana, Torr.,

Smilacina, Desf.,

S. racemosa, Desf.,

S. stellata, Desf.,

S. bifolia, Ker.,

Litium, L.

L. Canadense, L. var.

I. Washingtonianum, Kellogg, Erythionium, L.,

E. grandiflorum, Pursh.,

Frittillaria, L.

F. Kamtschatcensis,

F. lanceolata, Lindl.,

F. recurva, Benth.,

Litiorhiza, Kellogg.

L. lanceolata, Kellogg,
Three-leaved Nightshade.

False Hellebore.

False Asphodel.

False Solomon's Seal.

False Spikenard.

Lily.

White Lily.

Dog's Tooth Violet.

Twisted-Stalk.
Flag.

Hillsides.

Hillsides.

Woods.

Meadows.

Swamps.

Shady Places.

Redwoods.

Swampy Coast.

Moist Places.

Dry Hills and Plains.

Swamps.

Thickets.

Redwoods.

Damp Thickets.

Hillsides.

Redwoods.

Redwoods.

Thickets.

Shady Hillsides.

Shady Places.

Moist Places.

Hills.

Redwoods.

On Creeks.

Shady Hillsides.

Woods.

Meadows. 


\section{Latry Nimp:}

Cycukesing 1),

('. ailu, lioneh..

(' puhtheli.s. Rieneh..

cielegouss

C. intulea kiethen.

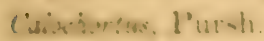

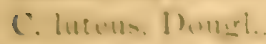

C. unithorus. Hhot of . Irn.. .

C. renustus. lieneh..

C'smensed. I.inll.

C. enuluentas limill.

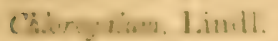

("i. pumet inlimum, liunth.. .

('). $n$.

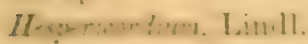

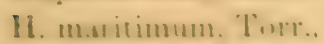

11 lac qeisu, linil ..

Bruthes. Sulth.

13. centerta. Kih..

13. enecentea limay.

13. -rrandiffera smieh,

13: Lrau liffora. vas ininor

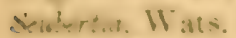

\$. Aaval kith.

(ia. !rem L. Lindll.

C. Intest I.ind1.

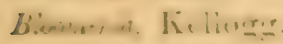

1. sureas livians.

filines. I...

A. iciuimaturn, Houk.,

A. falciolium. Hook.

A. amplectens, Torr. \& Gray ,

A. msritinum, Torr.,

A. ocvidentale, Gray.

Narlhecium, Moehring,

N. ossifragrum, var occidentale.,

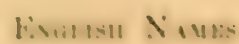

Naturar II agisat.

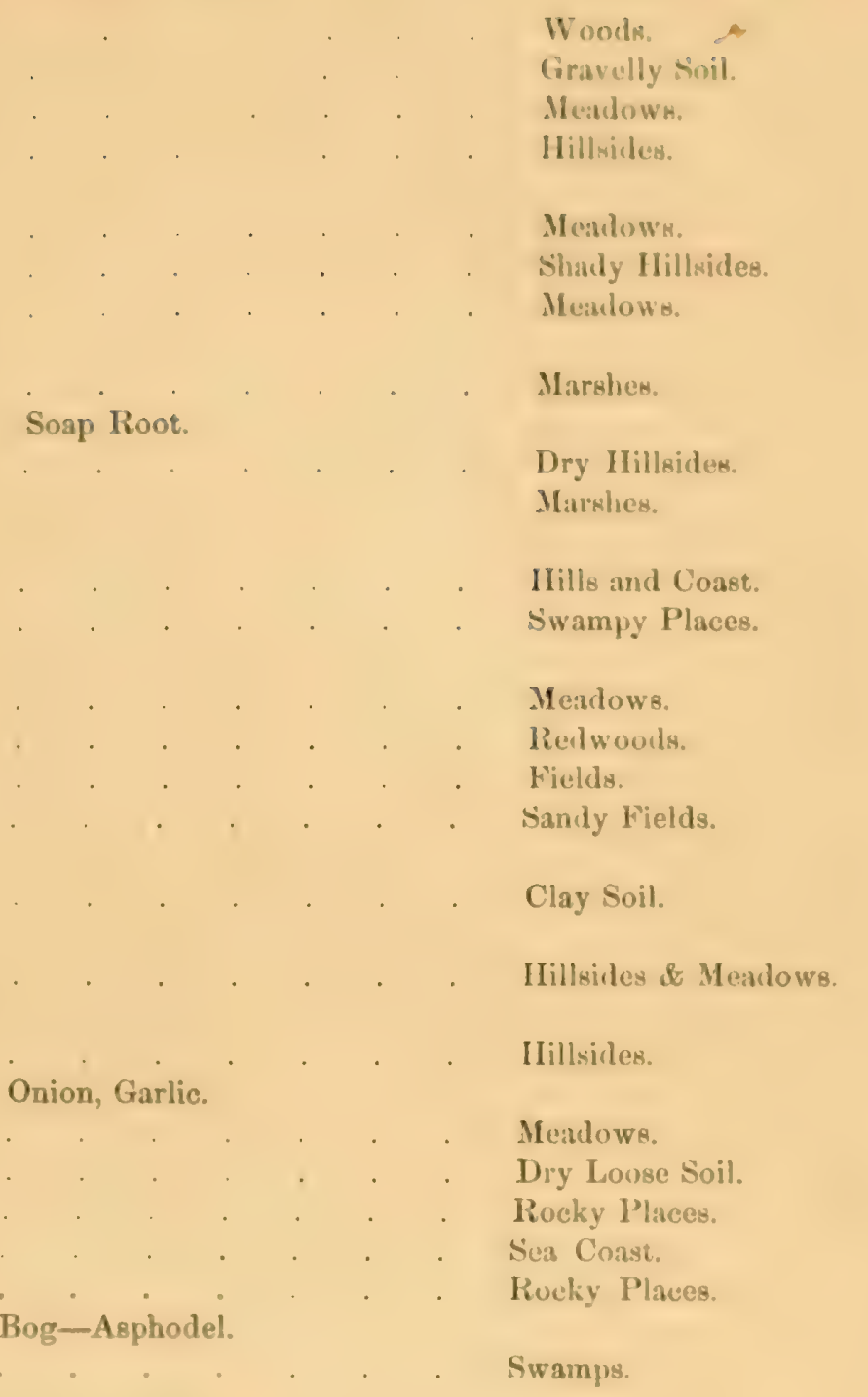

Juncacere. (Rueh Fumily.)

Leas? I).

1. rampuetrin. J1'

Jume. I...

J e.mprrents. HIt. \& K. .

J. lireweri. Fine...m.

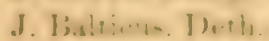

J $1, \ldots \ldots$ i $\quad[; \ldots 1$.

J. proweru- F. MI?

J. paten- F. MI:

J. Bripine I.

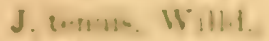

J. A. ..., E. Mथy.

J. cuponiform:- Engim
Wood Rush.

Bog Rush.
IIillsiles.

Sandy River Bottoms.

Wet Sandy Soil.

Markes.

Brackish Marshes.

sialt Mamshes.

Wet I'acen.

Everywhere.

Swampy Places.

Wet Mendowe.

Swampo. 
LATiN NAMES.

J. Bolanderi, Englm.,

J. xiphioides, E. Mey.

J. oxymeris, Englm.;

J. phaeocephalus, Englm., .

J. triformis, var. unitlorus, Englm.

J. Kelloggii, Englm.,
English Names.

Natural Habitat.

Wet Places.

Water Course.

Wet Places.

Wet Places.

Meadows.

Sandy Places.

Commelynaceae. (Spiderwort Family.)

Tradescantia, $\mathrm{L}$.

T. sp.

Oakland Hills.

\section{Cyperaceae. (Sedge Family.)}

Cyperus, I., .

C. erythrorhizos, Muhl.,

C. inflexus, Muhl.,

C. phymatodes, Muhl., .

Eleocharis, R. Br.

E. palustris, R. Br.,

E. sp.?

E. acicularis, $\mathrm{R}$. Br.,

Scirpus, L.,

S. pygmzus, Gray.,

S. carinatus, Gray.,

S. maritimus, L.,

S. sylvaticus, L.,

S. lacustris, L.,

S. criniger, Gray.,

S. triqueter. L.

Carex.,

C. siccata, Dewey,

C. Xalapensis, Kunth.,

C. Hookeriana, Dewey,

C. paniculata, L.,

C. leporina, L. ?

C. Deweyana, Schwartz,

C. propinqna, Nees.,

C. festiva, Dewey,

C. straminea, Willd.

C. lagapodioides, Schkr,

C. Hoodii, Boott,

C. steriis, Willd. var.,

C. stellulata, Good,

C. Bolanderi, Olney,

C. vulgaris, Fries var.*

C. salina Wahlbg var minor,

C. aquatilis, Wahlbr,

C. Sitchensis, Presc,

C. laciniata, Boott,

C. cryptocarpa, Mey.,

C. amplifolia, Boott,

C. Inxiflori, Iam. var. plantaginea, Boott,
Galingale.

Borders of Lakes.

Borders of Lakes.

Sandy River Banks.

Spike-Rush

Swamps.

Swamps.

Swamps.

Bulrush.

Salt Marshes.

Moist Meadows.

Sea Club-Rush.

Salt Marshes.

Swamps.

Water Courses.

Swamps.

Water Courses.

Sedge.

(Mark West's Creek.) Low Places.

(Mark West's Creek.) Swamps.

(Oakland.) Grassy Hillsides.

(Oakland.) Meadows.

Meadows.

(NapaValley.) Hillsides.

(Mark West's Creek.) Swamps.

(Punta de los Reyes.) Hillsides.

Meadows.

(Mark West's Creek.) Wet Places.

(Mark West's Creek.) Wet Places.

Swamps.

(Santa Rosa Creek.) Swamps.

(Redwoods.) Water Courses.

(Oakland.) Water Courses.

(Coast.) Swamps.

(Twelve Mile House.) Swamps.

(Tomales Bay.) Marshes.

(Mark West's Creek, Oakland.) Swamps.

(Twelve Mile House.) Swamps.

(Coast.) Cañons.

(Coast.) Dry Shady Hillsides. 
Latis Nave.

C. Kuieskernii, Dewey.

C. Eleluesa, limele.

C. (ieveri. Bonce.

C. tohtensora. Muls. var.

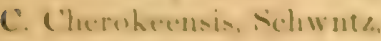

C. besuria, L。

C. utriculata, Buott,

C. Lystricina, Willd

C. polymorpha, Muhlbg., (New species.)

C. bitida, Boott,

C. gynodyвama, Olney,

C. cinnamomea, Oluey,

C. Luzulina. Olney,

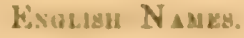

Natcrar Habitat.

Wot Places.

(Redwoods.) Hillsides.

(Rodwoods.) Hillsides.

Meaclows.

(Santa Rona Creek.) Swampa.

(Mark West's Creok.) Wet I'laces.

('Twelve Mile House.) swamps.

(Reiwoods.) Sw:mps.

Muadows.

(Salinas Valley.)

(Coast.) Meadows.

(Coast.) Mleadows.

(Coast.) Meadows.

Cramineae. Grases.

Lowric, solander,

L. oryzuidus, swartz,

Phalaris, L.

Ph. Canarieusis, L.

Ph. Californica, Hook.

Ph. angusta, Nees.,

Anthoranthem, L.

A. odorstum, $\mathbf{L}$.

Hrorochloa, Gmelin,

H. macrophylla, Thurb.

Holcus, L.

H. lanatus, I.

Beckmarnia, II ost.

B. crucselormis, Host.

Papalum, L.

P. distichum. L.

Panicum, L.

P. capillare, L.

P. dichoromum, L.

P. dichotomum, L. var. thermale Bol.

P. Crus-(ialli, L.,

Sitipa, 1 .

St. setigera, Presl.,? .

St. Phillipii, Stend. ?'

St. viridula, Trin.

Alopecurue, L.

A. pratensis,

A. Reniculatus, L.

Pheurn, L.

Ph. pratense, L.,

Ph. alpinum, L.

$V_{\text {slf }} a_{\text {, Adam, }}$

V. airciden, Torr.

Agrastio, L.

A. asperifolia, Trin.

A. scalsra, Willd., (laxifora)
White Grass.

Rice Cut Grase.

Canary Grass.

Canary Grass. (proper.) • . Fields.

Native Canary Grass. . . Moist Places.

Moist Meadows.

Sweet Verual Grass.

Holy Grass.

Meadow Soft Grass.

Velvet Grass.

Meadow日.

Redwoods.

Meadows.

Moist Meadows.

Joint Grass.

Moist Meadows.

Panic Grass.

Old Witch Grass. . . . Meadows.

Banks.

Geysers.

Barnyard Grass.

Along Streams.

F'eather Grass.

Hills and Meadows.

Hilisides and Thickets.

Bankm Eel River.

\section{Fox Tail Grass.}

Meadow Fox Tail.

Meadows.

Foating Fox Tail.

Moise Places.

Culis-tail Grass

Timothy.

Mesduws.

Fort Point.

Rush (irass.

Bent Grass.

Sundy Bauks.

Moist Places.

IIair Grase. . . . . Coast. 
Latin Names.

A. pallens, Trin.

A. exarata, Trin.,

A. canina, I.

A. pallida, DC, ??

A. vulgaris, With.

A. verticillata, Vill.

Polypogon, Desf.,

P. Monspeliensis, Desf.,

P. fugax, Nees.,

Vasaya, Thurb.

V. comata, Thurb. (Discovered by Dr. Kellogg, near Long Valley.)

Cinna, $\mathrm{L}$.

C. macroura, Kunth
English Names.

Natural Habitat.

Loose Soil

Everywhere.

Coast.

Moist Meadows.

Meadows.

Springy Places.

Beard Grass.

Springy Places.

Wet Places.

Rocky Places.

Dry Creeks.

* This grass is used by the Indians for making baskets,

Gastridium, Beauv.

G. australe, Beanv.

Hills and Hillsides.

* This specle covers all our bills late in the season. Introduced ?

Calamagrostis, Adans, .

C. sylvatica, DC,

C. Aleutica, Bong.

C. stricta, Tim.,

C. varia, Schrad.?

Phragmatis, Trin.,

P. communis, Trin.,

Cynodon, Rich.

C. Dactylon, Pers.

Spartina, Schr.,

S. stricta, Roth,

Arra, L.,

A. cespitosa, L.,

A. calycina, Presl. (danthonioides.) .

A. elongata, Hook.

Trisetum, Pers.,

T. cernuum, Trin.,

T. elatum, Nutt.,

T. barbatum, Steud.

Avena, $\mathrm{L}$.,

A. fatua, $\mathrm{L}$

Arrhenatherum, Beauv.

A. avenaceum, Beauv.

Danthonia, DC.,

D. Californica, Bol.,

Poa, $\mathrm{L}$.,

$P$. annua, $\mathrm{L}$.,

P. trivialis, L., .

P. pratensis, L.,

P. brachyphylla, Schult., .

P. Douglasii, Nees. (Californica, Steud., .
Reed Bent Grass.

Redwoods.

Hillsides.

Coast.

Moist Places Coast.

Reed.

Bermuda or Scoutch Grass.

Cord or Marsh Grass.

Salt Marsh Grass

Hair-Grass.

Common Hair Grass. . . . Wet Places.

Moist Shadows.

Moist Places.

Thickets.

Hillsides \& Meadows.

Hillsides \& Thickets.

Oat.

Wild-Oats.

Fields.

Oat Grass.

Oat Grass.

Meadow Grass, Speed Grass.

Low Speed Grass .

Ronghish Meadow Grass.

Common Meadow Grass.
Meadows.

Meadows.

Low Places.

Hillsides \& Meadows.

Meadows.

Moist Thickets.

Sandy Hills. 
Latrx Namx

Atruges liupr.

A. Colifurnie: Munro.

firceppormen. 1.inh

b. spitatum, llook of $\mathrm{L}$.

fir:an. I..

B. me lia.

Gi.

(i. famentina J'resl.

(i. nersata. Torr ..

(i. maritima. Wahl.

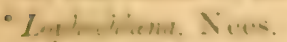

I. Calfirnica. Xers

M $\therefore$. 1...

M. imprereqa. Trin

M. protenilem. Nute.

M. (ieveri Munro.

II. acuminata, |3.,..

M Harfirdis. Isul.

hiven, l'ers.

K. rristata. l'ers.

Ino , i. I.

1) erinm.rata. I.

F : + I...

F. Iswrus. I...

F. wenella. Willi..

F. mi.rutaliss. Nutt.

F. oreiluntalis. Huok.,

F. seatrella, Torr.,

bir.menx. 1.

1).

B. sterilis. I...

B. ciliatus. I..

I) virels- Sintt.

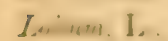

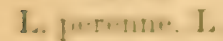

I. $\{$ เหm $\mid$ เntum, I.

\section{Tric.... I.}

T. ril, lis.

f., ... I.

E. Diluiricis, L.

E. condensatus, Presl.

Gymmoatuchum, Schreb.

G. Californicum. Bol.

Silonion. Raf.

S. elymoirles, $\mathbf{L}$.

II, i. $\mathrm{I}$..

II. $1+x^{+}+\ldots$ I... .

II. murit: in I.. .

Syture It. Hir.

J. Ris wathet, Thurh.

I. forkitals: : Nute.
ExuLıน Nasg.

Natukar Ilabitat.
. . . Meadows.

Spike Grass.

Quacking Grase.

Mamma Grass.

Fowl Mealow Grass. . . Water Courses.

Sea Spear Grass. . . . Salt Marshes.

Wet Meadows.

Melic-Grnss.

Orchard Grass.

Fescue Grass.

. . . Ficlds.

Ieadows.

Hillsicles.

Thickets.

Bunch Grass. . . . Moist Places.

Brome Grass.

Upright Chess.

Waste Places.

Waste Places.

Hillsides.

Moist Places \& Meadows.

Darnel.

live or Ray-Grass. . . Meadows.

bearded Darnel. . . . Grain Fields.

Wheat.

Quick-Grass.

Lime Grass.

Fields.

Fields \& Hillsides.

lBanks.

Open Woody Hillsides.

I)ry Hilkides.

Barley.

Siyuirrel-Tail Grase. . . . Mendows.

Waste I'laces.

I)ry IIillsides.

(13y Dr. Canfield, Montery.) . Sislinas I'lainu. 
Latin Names.

EnGLish Names.

Natural Habitat.

\section{CRYPTOGAMOUS OR FLOWERLESS PLANTS.}

Equisetacere. (Horse Tail Family.)

Equisetum, L.,

E. hyemale, $\mathrm{L}$,

E. eburneum, Schr.

E. fluviatile, L.
Scouring Rush.

Schave Grass. .

Banks.

Moist Thickets.

Shady Places.

Filices. Ferns.

Polypodium, L.

P. Californicum, Kaulf.

P. Scouleri, Hook.,

Adiantum, L.

A. pedatuma, $\mathrm{L}_{\text {。 }}$.

A. Chilense, Kaulf.

Petris, L.,

P. aquilina, L.,

Pellaea, Link.,

P. andromedæfolia, Hook,

P. mucronata, Eat.,

P. densa, Hook.

Gymnogramme, Desv.,

G. triangularis, Kaulf.

Woodwardia, Sm.

W. radicans, Willd,

Cystopteris Bernh.

C. fragilis, Bernh.

Aspidium, Sw.,

A. munitum, Kault.,

A. Californicum, Eat.,

Nephroduzm, Rich.,

N. rigidum, Desv.

N. Felix-mas, Rich.,

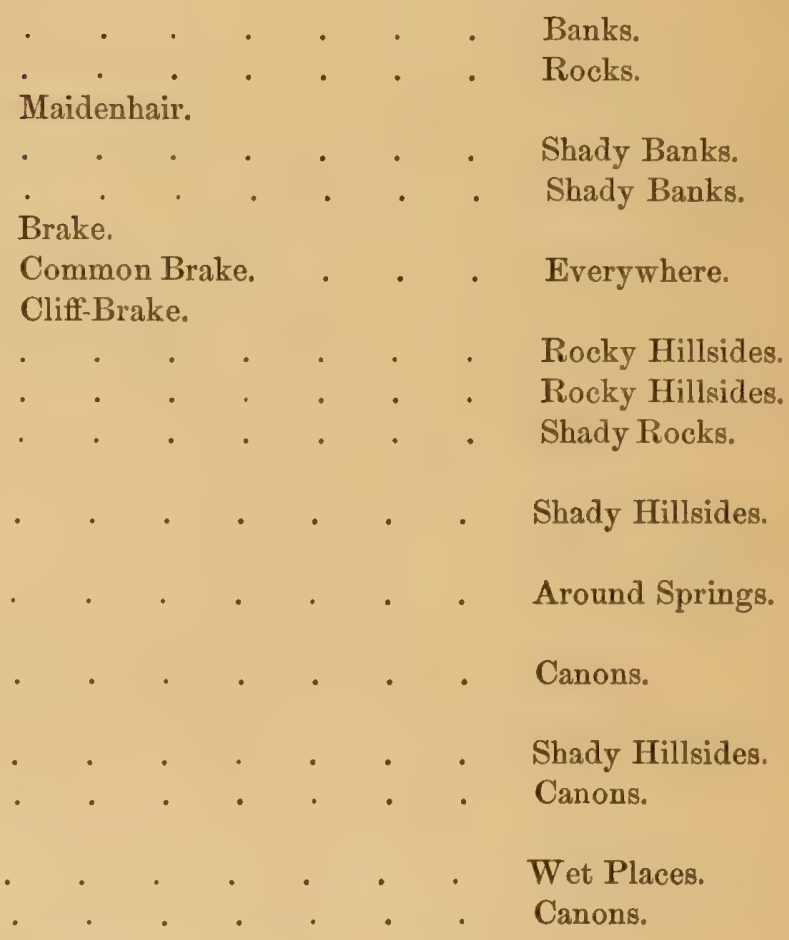

\section{Hydropterides.}

Azolla, Lam.,

A. Caroliniana.

Floating on Pools.

Musci. Mosses.

Sphagnum Dill.,

S. subsecundum, Nees \& Hrnsch,

Swamps-Coast.

S. subsecundum var. longifolium.

Swamps-Coast.

S. auriculatum, Schp., .

S. cymbifolium, Ehrh.,.

Ephemerum, Hpe.,

E. serratum, Hpe.,

Spherangium, Schp.,

S. muticum, Schp.,

Phascum, Schreb.,

Ph. cuspidatum, Schreb.,

Swamps-Coast.

Meadows.

Meadows.

Meadows. 


\section{LaTI Narias}

1'h. bryoidles, Dicks. .

Plewrudium, Brit.

I'. subulatum, Bruch of Sehpo,

Gymonostomum, lBreh \& sichp.,

G. calcarium, Nees ot Hrusch.,

Hoisio, Hedw:,

IV. viriclula, Bricl.,

W. oirrhata, Hedw.

\section{I)}

D. varium, Hedw.

D. heteromallum, Hedw.

D. Aimench- Tarn...

1). - ...

$1 \ldots, 11,1 w$.

F. limbin-siol.

F.endimens liris..

A : : lin. li.

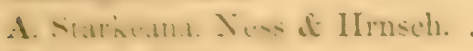

(…... Init.

C. purpurens, Brid., .

$I$ Hpe.

L. Sulhimperi, Lesy.,

Trichosiomun, Hedw.

T. rigiclulum, Snith., .

T. tophaceum, Brid.,

F. anomalum. Schp., .

F. Alexipes, Bruch \& Schp.

Dermatodon, Brid.,

D. Californicus, Lesqx.

D. Guepini, Bruch \& Schap.,

Barbula, IIedw.

B. fallax, Heclro.,

B. subfallax, Mull.,

B. artocarpa, Lesqx., .

B. vinealis, Brid.,

B. flexifolis, Ipe,

B. Virescens, Lesqx., 。

B. convoluta. Hedw.

B. cuneifolia, Brid.,

B. Wahlians, Schultz.

B. Bolanderi, Lesyx.,

B. amplera, Lesqx。

B. marginata, Bryol., Eu.,

B) hrovip- 1..... A

b. inermi- linuts.

If Heripta Br.u.

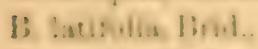

18. curail- He. 3 w

13. Maellen limb.

Giremes, Fitit

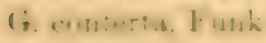

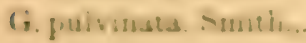

\section{Evousm Nayma.}

Niteiral II hutat.

Grasy Ilillsides.

Borders of Ditches.

Wet Rocks.

Sharly Hillsides.

On Renlwoods.

Borders of C'reeks.

Ditches.

Redwood s'tumps.

Redwood stumps.

Shady IIillsides.

Wet Rucks.

Clay Soil.

IIillsides.

Dry Borders.

Ground.

Wet Rocks.

Ground.

Ground.

Shady Borders.

Shady Borders.

Rocks.

Rocks.

Borders of Creeks.

Sandy Soil.

Rocks.

Ground.

Shady Ground.

Shady Ground.

Shady Ground.

Rocks.

Shady Ground.

Ground.

limk:

linchs.

lisuhe.

Isuriles of ( 'reeks.

Iinkh.

Iinche

Runckis.

Ruclis. 
Latin Names.

ENGLish Names.

Natural Habitat.

G. trichophylla, Grev.,

G. Californica, Sullv.,

G. leucophæa, Grev.,

G. montana, Brch \& Schp.,

Racomitrium, Brid.,

R. canescens, Brid.,

Hedrorgia, Ehrh.

H. ciliata, Ehrh.

Braunia, Brch \& Schp.,

B. Californica, Lesqx.,

Zygodon, Bruch \& Schp.,

Z. Californicus, Muell., .

Orthotrichum, Hedw.,

O. cupulatum, Hoffm.,

O. Texanum, Sull.,

O. speciosum, Nees.,

O. rivulare, Turn.. .

o. cylindrocarpum, Lesq $x_{9}$

O. Lyellii, Hook,

O. pulchellum, Smith,

Encalypta, Schreb.

E. vulgaris, Hedw.,

E. ciliata, Hedw.,

Physcomitrium, Brid.

P. pyriforme, Brid.,

Enthostodon, Schwægr.

E. Bolanderi, Lesqx.,

E. Templetoni, Schw.,

Funaria. Schreb.

F. Californica, Sull \& Lesqx.,

F. calcarea, Wahl,

F. hygrometrica, Hedw.,

Bryum, Dill.

B. pyriforme, Hedw.,

B. Tozeri, Grev.,

B. albicans, Wahl.,

B. torquescens, Bruch \& Schp.,

B. argenteum, L., .

B. Californicum, Sull.,.

B. occidentale, Sull.,

B. Canariense, Brid.,

Mnium, L.

M. affine, Bland,

M. venustum, Mitt,

Autacomnium, Schwgr.

A. androgynum, Schwgr.

A. palustre, Schwgr.,

Bartramia, Hedw.

B. stricta, Brid.,

B. fontana, Brid.,

B. Menziesii, Turn., .

Atrichum, Tal. Beauv.

Rocks

Rocks.

Rocks.

Rocks.

Rocks.

Rocks.

Rocks.

Shaded Rocks.

Trees.

Trees.

Trees.

Trees.

Trees.

Trees and Rocks.

Trees.

Ground.

Shaded Rocks.

Swampy Places.

Banks.

Gravelly Soil.

Gravelly Soil.

Soil.

Everywhere.

Wet Borders.

Wet Borders.

Hillsides.

Hillsides.

Hillsides.

Hillsides.

Hillsides.

Hillsides.

Shady Banks.

Shady Places.

Redwood Stumps.

Swamps.

Ground.

Springy Places.

Rocks. 
A. umelulatum, Tal. lie:auv.

Polyorbinum, Dill.

P. juniperinum, Hedw。

Rocky Ground.

Fontinalis, Dill.

F. antip! rutien.

Sitrenus.

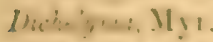

1). Sw:trii, limill.

Swamp*.

Jienar. Helw.

N. Menziesii, Hook, .

T'rees.

N. Douglasii, Hook.,

'Trees. Alsio, sull.

A. Californica, Sull..

Rucks of 'Trees.

A. longipes, Sull \& Lesqx.,

lineks.

A. abietina, sull.

Antitrithio, Brid.,

A. curtipenduia, var. gigantea, Sull \& Lesqx., .

A. Caitornies, sull., Anomodon, Hook ot Tayl.

A. Californicum, Lesqu., .

Fabronia, Ratdi.

F. pusilla, Ratui.

Plenogonimm, Swartz.

P.gracile, Swartz, .

Вурими, Dill.

H. crispifolium. Hook,

H. leuconeurum, Sull \& Lesqx.,

H. myosuroides, L.,

H. stoloniferum, Hook,

H. Brewerianum, Lesqq.,

H. aggregatum, Mitt.,

H. Nuttallii. Wils., .

H. arenarium, Lesqx.,

H. pinnatialum, Sull d Lesqx.,

H. vallium, Sull \& Lesqx.,

H. Bolanderi, Iesy $x$.,

H. illecebrum, Schwagr. .

H. cespitosum, Wils.

H. Calitornicum, Lesqx.,

H. Stockesii. 'Turn., .

H. Oreganum. Sull.,

H. rusciforme. Weis,

H. Bigelovii, Sull,

H. serpens, L.

H. riparium, L.,

H. aduncum. Heilw:,

H. subimponeus, Lesqx. .

H. circinale. Hook.

Hepaticae. (Livereorts.)

Sub-Order I. Ricciacea.

Riccia. Mich.

R. glanea L.

Borilers of IDitches.

Rocks.

liveks \& 'Trees.

Ground.

Trees.

Rocks \& Trees.

Rocks.

Ground.

Shated Rocks.

Shaded Rocks.

shiaded Rocks.

Shaded Rocks.

Trees.

Sandy Soil.

Rocks.

Streams.

Shady Ground.

Shady Ground.

Shady Ground.

Trees.

Borders of Streams.

Rocks \& 'Trees.

Wet Iiocks.

Shady Wet Borders.

Willow Swamps.

Borders.

Swamps.

Rocks \& Stumps.

Realwood Stumps. 
Latrin Names.

R. nigrella,

R. lamellosa, Raddi.,

R. crystallina, L.

Spharocarpus, Mich.,

S. Michelli, Bellard,

Anthoceros, Mich.

A. punctatus, $\mathbf{L}$.

A. lævis, L.,
English Names.

Round Headed Liverwort.

\section{Sub-Order II. Authocerotea.}

Horned Liverwort.

Shady Banks.

Shady Banks.

Gravelly Soil.

Gravelly Soil.

Gravelly Soil.

Clay Soil.

\section{Sub-Order III. Marchantiacea.}

Targionia, Michel,

T. Michrlii, Corda.,

Marchantia, L.,

M. polymorpha, L.,

Brook Liverwort.

Moist Shady Rocks \& Banks.

Grimaldia, Raddi.

G. Californica, Gottsche,

Fimbriarla, Nees.,

F. tenella, Nees.,

F. Bolanderi, Austin,

F. Californica, Hpe (Lescurii Austin)

Natural Habitat.

\section{Brook Liverwort.}

Wet Places.

Banks.

Small Liverwort.

Moist Shady Hillsides.

Banks.

Around Rocks.

Sub-Order IV. Jungermauniacoze. (Scale Mosses.)

Aneura, Dum.

A. pinguis, Dum.,

A. palmata, Nees., Pellia, Raddi.

P. calycina, Taylor,

Fossombroma, Raddi.

F. pusilla, Nees.,

Jungermannia, L.,

J. rubra, Gott,

J. biscuspidata, L.

Scapania, Lind.

S. undulata, Nees.,

S. Californica, Gott,

Sarcoscyphus, Corda.

S. Francisci, Gott,

Frullania, Raddi.

F. Petalumensis, Gott,

F. unciflora, b. Californica, Gott,

F. Bolanderi, Aust.,

F. Tamarisci, Nees.,?

Madotheca, Dum.,

M. rivularis, Gott,

M. platyphy]la, Dum.,

Lepidosia, Nees.,

L. reptans, Nees.,

Radula, Nees.

R. complanata, Dum.,
Shady Banks.

Shady Banks.

Shady Banks.

Wet Shady Banks.

Scale Moss.

Rocks.

Swamps.

Trees.

Trees.

Stumps.

Bark.

Bark.

Bark.

Rocks.

Tree Scale Moss.

Trees.

Trees \& Banks.

Creeping Scale Moss.

Stumps.

Bark. 
LATiN Nanes.

Evolistr NaMrs.

Lichenes. (Lichens.)

\section{Tribe 1. Parmeliacel, Fr.}

Ramelims, Ach.

R. Menriesii, Tayl.,

R. homalea, Ach.,

R. ceruchis, Ach.,

Cotromia, Ach.

C. Californica, Tuck.,

C. glaues, Ach.,

C. lacunosa, Ach..

C. ciliaris, Ach.,

C. juniperina, Ach.

Enervia, Ach.

E. vulpina, Ach.,

E. prunastri, Ach.

Conea, Hottim.

$\mathbb{U}$. barbata, var.

Thelosclustes.

Th. chrysophthalmus,

Th. parietinus, L. var Finmarkicus, Ach.,

Parmelia, Fr.

P. perlata, Ach., var flavicans,

P. perforata, Ach.

P. saxatilis, Fr.,

P. physodes, Ach.

P. olivaces, Ach.

P. caperata, Ach.,

Physcia, schrbr.

$\mathrm{Ph}$. erinacea, Tuck.

Ph. speciosa, TVulf.

$\mathrm{Ph}$. stellaris, L.

Cmbilicaria, Hoffim.

C. phaca, Tuck.,

U. hirsuta. DC.,

U. angulata. Tuck..

Stscla, Schreb.

H. fuliginosa, Ach:

H. anthraspis, Ach..

H. pulmonaria, Ach.,

H. scorbiculata. Ach.

Pelligera, Willd.

P. aphthosa, Hotim.

P. canina, Hoffm.,

P. rufescens, Hoffm.,

P. polyilactyia, Hoff,

P. venosa. Hotlim.

Pannaria.

P. microphylla, Massal,

P. lepiliota, Fr.

P. cyanulepra, Tuck.
Natuhar II amtat.
Trees \& Fences.

Rocks at the Coast.

Trees.

Fences \& Trees.

Trees \& Coast.

l'ine Trees.

Trees.

Pine Trees.

Trees.

Trees.

Trees.

Fences.

Rocks.

Rocks.

Rocks.

Rocks.

Trees.

Trees.

Rocks.

Wood.

Rocks.

Rocks.

Rocks.

Mossy Rocks.

Oak Trees \& Rocks.

Trees.

Iossy Rocks.

Giounil.

Iossy Rocks.

Trees \& Rocks.

Trees \& (iround.

Mrssy Grouni.

Mossy Rocks.

Mosay Rocks.

(iround. 
Latin Names.

English Names.

Natural Habitat.

Dirina, Fr.

D. Californica, Tuck.,

Oak Trees.

Placodium.

P. coralloides, Tuck.,

P. callopismum, Merat,

P. bolacinum, Tuck.,

P. cinnabarrinum, Anz.,

P. vitellinum, Ehrh.,

Lecanora, Ach.

L. Bolanderi, Tuck.

L. phryganitis, Tuck.,

L. thamnitis, Tuck.,

L. muralis, Schreb.,

L. pinguis, Tuck.,

I. varia, Ach.,

I. pallescens, Schær.,

L. pallida, Shær.,

I. glaucoma, Ach.,

L. Cenisia. Ach.,

L. subfusca, Ach.,

L. atra, Ach.,

L. fecunda, Tuck.,

L. cinerea, Sommerf.

L. Scheicheri, Nyl.,

L. xanthophana, Nyl.,

L. rubina, var opaca,

L. Brunonis, Tuck.,

Rinodina,

R. sophodes, Ach.,

R. sabulosa, Tuck,

Pertusaria, DC.

P. pertusa, Ach.,

P. Wulfenii, Nyl.,

P. velata, var,

Urceolaria, Ach.

U. scruposa, Ach.,

Rocks.

Rocks.

Rocks.

Rocks.

Rocks.

Rocks.

Rocks.

Rocks.

Rocks.

Rocks.

Rocks.

Trees.

Trees \& Rocks.

Rocks.

Rocks.

Rocks.

Rocks.

Trees.

Rocks.

Wood.

Rocks.

Pines.

Rocks.

Trees.

Ground.

Trees.

Trees.

Oak Trees.

Ground.

\section{Tribe II. Lecideacei, Fr.}

Stereocaulon, Ach.

St. paschale, 'Tr.

Cladonza, Hoffm.

C. Santensis, Tuck.,

C. pyxidata, Fr.

C. gracilis, Fr.

C. flmbriata, Fr.,

C. bellidiflora, Schær.

Biatora, Fr.

B. globifera, Fr.,

B. decipiens, Fr.

B. scotopholis, Tuck.,

B. coarctata, $\mathrm{Fr}$.,

B. glebulosa, Fr.

Ground.

Ground.

Ground.

Ground.

Ground.

Ground \& Trees.

Rocky Soil.

Rocky Soil.

Rocky Soil.

Rocky Soil.

Rocky Soil. 
Lecidea, Ach.

L. enteroleuca, Ach.,

L. insularis, $\mathrm{Nyl}$.

L. ruginosa, Tuck.,

L. massata, Tuck.

Buellia.

B. radiata, Tuck.,

B. Bolanderi, Tuck.,

B. lactea, Kerb,

B. stellulata, Tayl.,

B. pullata, Tuck.,

- B. halonia Ach.

B parasema, Kerb.,

B. parasitica, Fr.

B. oidalea, Tuck.

B. geographica L.,

Rocks.

Rocks.

Rocks.

Gravelly Soil.

Rocks \& Coast.

Rocks.

Rocks.

Rocks.

Rocks.

Rocks.

Rocks.

Rocks.

Trees.

Rocks.

Tribe III. Graphidacei, Nyl.

Opegrapha, Pers.,

O. varia, Fr.,

O. vulgata, Nyl., .

Sphacrophorus, Ach., S. globiferus, DC., Acolsum.,

A. Californicum, 'Tuck.

A. Bolanderi, Tuok.,

A. chloroconium, Tuck.

A. tigillare, Fr.,

Calicium, Pers.,

C. subtile, Fr.

C. chrysocephalum, Ach.

C. hyperellum, Wahl., .

Trees.

Trees.

\section{Tribe IV. Caliciacei.}

Stumps.

Rocks.

Rocks.

Trees.

Trees.

Pines.

Pines.

Pines.

\section{Tribe v. Verrucariacei, Fr.}

Dermatocarpon.

D. mineatum, Eschro.,

D. Guepini, Moag.,

Collema, Ach.,

C. nigrescens, Ach.,

C. coccophorum.

Loptogium, Fr.,

L. palmatum, Mont.,

L. muscicola, Fr.

L. albociliatum, Tuck.,

L. Californicnm, Tuek.

Ground.

Ground.

Trees.

Ground.

On Mosses.

On Mosses.

Rocks.

Rocks. 


